Pacific Journal of Mathematic 


\title{
ON THE STABILITY OF BOUNDARY COMPONENTS
}

\author{
KÔTARO OIKAWA
}

\section{Presentation of the Problem}

\section{Definitions.}

1. A boundary component of a plane region $D \subset(|z| \leqq \infty)$ is a component of the boundary $\partial D$ of $D$, i.e., a connected subset of $\partial D$ which is not a proper subset of any connected subset of $\partial D$.

There is an alternate definition. Let $\left\{\Omega_{n}\right\}_{n=1}^{\infty}$ be a sequence of subregions of $D$ such that

(i) $\Omega_{1} \supset \Omega_{2} \supset \cdots$,

(ii) the relative boundary $\partial \Omega_{n} \cap D$ consists of one closed analytic curve in $D$,

(iii) $\bigcap_{n=1}^{\infty} \Omega_{n}=\phi$. Two sequences $\left\{\Omega_{n}\right\}$ and $\left\{\Omega_{n}^{\prime}\right\}$ are said to be equivalent if, for any $n$, there exists $m$ such that $\Omega_{m} \subset \Omega_{n}^{\prime}$ and $\Omega_{m}^{\prime} \subset \Omega_{n}$. A boundary component of $D$ is an equivalence class of $\left\{\Omega_{n}\right\}$.

These two definitions are equivalent in the following sense:

(i ) Given a sequence $\left\{\Omega_{n}\right\}$, the set $\bigcap_{n=1}^{\infty} \bar{\Omega}_{n}$ is a component of $\partial D$ and, for two sequences, these sets coincide if and only if the sequences are equivalent.

(ii) Given a component $\Gamma$ of $\partial D$, there exists a sequence such that $\Gamma=\bigcap_{n=1}^{\infty} \bar{\Omega}_{n}$.

For a boundary component $\Gamma$, the sequence $\left\{\Omega_{n}\right\}$ such that $\Gamma=\bigcap_{n=1}^{\infty} \bar{\Omega}_{n}$ is called a defining sequence of $\Gamma$.

Let $w=f(z)$ be a topological mapping of $D$ onto a plane region $D^{\prime}$. Then we can immediately see from the second definition that $f$ gives a one-to-one correspondence between the boundary components of $D$ and $D^{\prime}$. We shall speak of the image of a boundary component $\Gamma$ under $f$ in this sense and denote it by $f(\Gamma)$.

2. Let $D^{c}$ denote the complement of $D$ with respect to the extended plane $|z| \leqq \infty$. For a boundary component $\Gamma$, there exists a uniquely determined component of $D^{c}$ whose boundary coincides with $\Gamma$. We call it the component of $D^{c}$ corresponding to $\Gamma$ and denote it by $\Gamma^{*}$.

If $D$ does not contain the point $z=\infty$, the boundary component $\Gamma$

Received January 7, 1959. The present paper is a part of the author's doctoral dissertation submitted to the University of California, Los Angeles. The author wishes to express his heartiest gratitude to Professor Leo Sario for his guidance and encouragement during the preparation of this paper. 
such that $\infty \in \Gamma^{*}$ is called the outer boundary of $D$.

3. We call a region $D$ a circular (or radial) slit disk if $0 \in D$, $D \subset(|z|<R<\infty)$, the outer boundary is $|z|=R$, and every other boundary component is either a point or an arc on $|z|=$ const. (or a line segment on $\arg z=$ const.).

\section{The stability problem of boundary components.}

4. Let $D$ be a plane region and let $\Gamma$ be a boundary component. Sario $[16,17]$ gave the following classification:

(a) If $f(\Gamma)$ is a point for every univalent function $w=f(z)$ on $D$, then $\Gamma$ is said to be weak.

(b) If $f(\Gamma)$ is a continuum, i.e., a connected closed set containing more than one point, for every $f$, then $\Gamma$ is said to be strong.

(c) If $\Gamma$ is neither weak nor strong, it is said to be unstable.

Weak boundary components were first investigated by Grötzsch in connection with the so-called "Kreisnormierungsproblem" (Grötzsch [7]; see also Denneberg [5] and Strebel [21]). He called them vollkommen punktförmig. Regions of class $O_{S B}=O_{S D}$ introduced by Ahlfors and Beurling [2] coincide with those possessing merely weak boundary components. Sario [16] has generalized the concept weak boundary components for open Riemann surfaces. It has been discussed also by Savage [19] and Jurchescu [10].

We are now lead to the following natural problems:

Problem A. Given a boundary component consisting of a single point, determine whether it is weak or unstable.

Problem B. Given a boundary component consisting of a continuum, determine whether it is strong or unstable.

We shall attempt to obtain concrete tests with practical applicability.

\section{Related extremal problems.}

5. Let $D$ be a region containing the point $z=0$. Let $\mathfrak{B}$ be the family consisting of all functions $w=\phi(z)$ which are regular and univalent in $D-\{0\}$, and have the expansion $1 / z+c z+\cdots$ near $z=0$.

Consider, with Grötzsch [6], the diameter of the image $\varphi(\Gamma)$ of the boundary component $\Gamma$. It is quite easy to see that $\Gamma$ is weak if and only if $\sup _{\varphi \in \mathfrak{B}} \operatorname{diam} \varphi(\Gamma)=0$, and $\Gamma$ is strong if $\inf _{\varphi \in \mathfrak{B}} \operatorname{diam} \varphi(\Gamma)>0$.

6. Let $\mathfrak{F}_{r}$ be the family consisting of functions $w=f(z)$ such that

(i) regular and univalent in $D$,

(ii) $f(0)=0$ and $f^{\prime}(0)=1$, 
(iii) $f(\Gamma)$ is the outer boundary of $f(D)$.

Rengel [14] introduced the following functionals on $\widetilde{F}_{\Gamma}$ :

$$
\begin{aligned}
& M(f)=\max _{w \in f\left(\Gamma^{\prime}\right)}|w|=\sup _{z \in D}|f(z)|, \\
& m(f)=\min _{w \in f(\Gamma)}|w|,
\end{aligned}
$$

and considered the quantities

$$
R(\Gamma)=R(\Gamma ; D)=\sup _{f \in \mathfrak{F} \Gamma} m(f)
$$

and

$$
r(\Gamma)=r(\Gamma ; D)=\inf _{f \in \mathfrak{F}_{\Gamma}} M(f) .
$$

From the definition we have immediately the basic

Theorem 1. $\Gamma$ is strong if $R(\Gamma)<\infty . \quad \Gamma$ is weak if and only if $r(\Gamma)=\infty$.

These criteria are equivalent to those in No. 5 , since

$$
\begin{aligned}
R(\Gamma) & =2 / \inf _{\varphi \in \mathfrak{B}} \operatorname{diam} \varphi(\Gamma), \\
r(\Gamma) & =4 / \sup _{\varphi \in \mathfrak{B}} \operatorname{diam} \varphi(\Gamma) .
\end{aligned}
$$

In fact, for an arbitrary function $f(z) \equiv \mathfrak{F}_{\Gamma}$, the functions

$$
\varphi_{f}(z)=\frac{1}{f(z)}+\frac{f^{\prime \prime}(0)}{2}
$$

and

$$
\psi_{f}(z)=\varphi_{f}(z)+\frac{1}{M(f)^{2}} \cdot \frac{1}{\varphi_{f}(z)}
$$

belong to $\mathfrak{B}$, and

$$
\begin{aligned}
& m(f) \leqq 2 / \operatorname{diam} \varphi_{f}(\Gamma) \\
& M(f) \geqq 4 / \operatorname{diam} \varphi_{f}(\Gamma) .
\end{aligned}
$$

On the other hand, for $\varphi(z) \in \mathfrak{B}$, let $F(w)$ be the function which maps $\left(\varphi(\Gamma)^{*}\right)^{c}$ conformally onto the exterior of a disk with the center at the origin. Assume further that $F(w)=w+c+c^{\prime} / w+\cdots$ near $w=\infty$. Then $f_{\varphi}(z)=1 / F \circ \varphi(z) \in \widetilde{F}_{r}$ and

$$
2 / \operatorname{diam} \varphi(\Gamma) \leqq M\left(f_{\varphi}\right)=m\left(f_{\varphi}\right) \leqq 4 / \operatorname{diam} \varphi(\Gamma) .
$$


The proof of the above equalities is hereby complete.

7. Whether or not $R(\Gamma)<\infty$ is necessary for strength is still an open problem. We shall discuss this problem in No. 24.

We shall see in No. 17 that $1 / r(\Gamma)$ equals the "capacity" of the boundary component $\Gamma$ introduced by Sario [16] (it is not necessarily equal to the logarithmic capacity of the closed set $\Gamma$ ), and, therefore, that the latter half of Theorem 1 is equivalent to Sario's result ([17], Theorem 6). Jurchescu [10] showed that the "capacity" coincides with the "perimeter" introduced by Ahlfors and Beurling [2].

It will be shown in No. 22 that $R(\Gamma)$ coincides with the quantity which Strebel [22] called "extremal Durchmesser". Finally, Theorem 4 in No. 21 shows that the first half of the above theorem coincides with Sario's result ([17], Theorem 4).

\section{Preliminaries}

In this chapter, we collect a number of known results which will be needed later.

\section{Extremal length.}

8. A curve $\gamma$ considered here is either a closed rectifiable curve or a curve of the form $z=z(t) \quad(0<t<1)$ every subarc of which is rectifiable. If $\lim _{t \rightarrow 0} z(t)$ or $\lim _{t \rightarrow 1} z(t)$ exists, it is called an end point.

Let $D$ be a reginon and let $\{\gamma\}$ be a family of curves $\gamma \subset D$. Let $\{\rho\}$ be the collection of functions $\rho$ which are $\geqq 0$ and lower semi-continuous in $D$. With the understanding that $0 / 0=\infty / \infty=0$, take

$$
\lambda\{\gamma\}=\sup _{\rho} \frac{\left(\inf _{\gamma} \int_{\gamma} \rho d s\right)^{2}}{\iint_{D} \rho^{2} d x d y} .
$$

It is called the extremal length of $\{\gamma\}$ (Ahlfors and Beurling [2], Ahlfors and Sario [3]).

9. The following properties (I)-(V) are well known; for the proofs the reader is referred to, e.g., Hersch $[8]^{1}$ :

(I ) $\lambda\{\gamma\}$ is independent of the choice of $D$.

(II) $\lambda\{\gamma\}$ is conformally invariant.

(III) $\lambda\left\{\gamma^{\prime}\right\} \leqq \lambda\{\gamma\}$ if every $\gamma$ contains a $\gamma^{\prime}$.

(IV) For $\left\{\gamma_{1}\right\}$ and $\left\{\gamma_{2}\right\}$, assume the existence of disjoint regions $D_{1}$ and $D_{2}$ such that $\gamma_{\nu} \subset D_{\nu}(\nu=1,2)$. If, for any $\gamma$ of the third family

\footnotetext{
${ }^{1}$ His definition is different from ours, but his proofs remain valid.
} 
$\{\gamma\}$, there exist $\gamma_{1}$ and $\gamma_{2}$ such that $\gamma_{1} \cup \gamma_{2} \subset \gamma$, then

$$
\lambda\left\{\gamma_{1}\right\}+\lambda\left\{\gamma_{2}\right\} \leqq \lambda\{\gamma\} \text {. }
$$
then

(V) Let $\left\{\gamma_{1}\right\}$ and $\left\{\gamma_{2}\right\}$ be the same as above. If $\left\{\gamma_{1}\right\} \cup\left\{\gamma_{2}\right\} \subset\{\gamma\}$,

$$
\frac{1}{\lambda\left\{\gamma_{1}\right\}}+\frac{1}{\lambda\left\{\gamma_{2}\right\}} \leqq \frac{1}{\lambda\{\gamma\}}
$$

(VI) (Hersch [8] $]^{1}$. For three families with $\{\gamma\}=\left\{\gamma_{1}\right\} \cup\left\{\gamma_{2}\right\}$,

$$
\frac{1}{\lambda\{\gamma\}} \leqq \frac{1}{\lambda\left\{\gamma_{1}\right\}}+\frac{1}{\lambda\left\{\gamma_{2}\right\}}
$$

(VIII) Let $\left\{\gamma_{1}\right\}$ be the subfamily of $\{\gamma\}$ consisting of $\gamma$ having both end points and such that $z(t)(0 \leqq t \leqq 1)$ is rectifiable. Then $\lambda\{\gamma\}=\lambda\left\{\gamma_{1}\right\}$. In fact, since the extremal length of $\left\{\gamma_{2}\right\}=\{\gamma\}-\left\{\gamma_{1}\right\}$ is infinite, (VI) shows that $\lambda\left\{\gamma_{1}\right\} \leqq \lambda\{\gamma\}$, and $\lambda\{\gamma\} \leqq \lambda\left\{\gamma_{1}\right\}$ by (III).

(VIII) For a curve $\gamma: z=z(t) \quad(0<t<1)$, let $\bar{\gamma}$ be the curve $z=\overline{z(t)}(0<t<1)$. If $z(0)=\lim _{t \rightarrow 0} z(t)$ exists and is real, put $\hat{\gamma}=$ $\gamma \cup \bar{\gamma} \cup\{z(0)\}$. Let $\left\{\gamma_{0}\right\}$ be a family of curves which are contained in the upper half-plane and have the end points $z(0)$ on the real axis. Let $\{\gamma\}$ be a family which contains all $\hat{\gamma}_{0}$ and $\bar{\gamma}$. Furthermore it is assumed that, for any $\gamma$, there exist $\gamma_{0}$ and $\gamma_{0}^{\prime}$ in $\left\{\gamma_{0}\right\}$ such that $\bar{\gamma}_{0} \cup \gamma_{0}^{\prime} \subset \gamma$. Then

$$
\lambda\{\gamma\}=2 \lambda\left\{\gamma_{0}\right\}
$$

In fact, to define $\lambda\{\gamma\}$, we may restrict $\{\rho\}$ to the subfamily consisting of functions symmetric about the real axis. Since $2 \inf _{\gamma_{0}} \int_{\gamma_{0}} \rho d s=$ $\inf _{\gamma} \int_{\gamma} \rho d s$ for such $\rho$, we conclude that $\lambda\{\gamma\}=2 \lambda\left\{\gamma_{0}\right\}$.

(IX) Let $A$ be the annulus $1<|z|<q$ or a region obtained by deleting a finite number of circular slits from this annulus. Let $\{\gamma\}$ be the family of all closed rectifiable curves in $A$ separating $|z|=1$ from $|z|=q$. Then $\lambda\{\gamma\}=2 \pi / \log q$. This is true even if each $\gamma$ is restricted to a concentric circle in $A$.

The proof is found, e.g., in Hersch [8] 1 .

10. Let $D$ be a region, and let $E_{0}$ and $E_{1}$ be compact sets such that $E, \cap \bar{D} \neq \phi(\nu=0,1)$. Let $\{\gamma\}$ be the family consisting of $\gamma$ : $z=z(t)(0<t<1)$ such that $\gamma \subset D, \bigcap_{\varepsilon>0} \overline{\{z(t) ; 0<t<\varepsilon\}} \subset E_{0}$, and $\bigcap_{\varepsilon>0}\{\overline{\{z(t) ; 1-\varepsilon<t<1}\} \subset E_{1}$. Then $\lambda\{\gamma\}$ is called the extremal distance $\delta_{D}\left(E_{0}, E_{1}\right)$ between $E_{0}$ and $E_{1}$ with respect to $D$.

By (VII), $\delta_{D}\left(E_{0}, E_{1}\right)$ coincides with the extremal length of the family 
of rectifiable curves in $D$ whose end points are on $E_{0}$ and $E_{1}$ respectively. Under a certain restriction of the configuration, it is also equal to that of a subfamily consisting of analytic curves (Wolontis [25]).

From this consideration, we get

(X) If no point of $E_{1}$ is accessible from $D$ by a rectifiable curve, then $\delta_{D}\left(E_{0}, E_{1}\right)=\infty$.

(XI) (Pfluger [12] $]^{1}$ ). If cap $E_{1}=0$, then $\delta_{D}\left(E_{0}, E_{1}\right)=\infty$. For $D=(|z|=1), \quad E_{0}=(|z|=\varepsilon<1)$, and $E_{1} \subset(|z|=1), \quad \delta_{D}\left(E_{0}, E_{1}\right)=\infty$ if and only if cap $E_{1}=0$.

Combining (VI), (X), and (XI), we get

(X') If no point on $E_{1}$, except for a set of capacity zero, is accessible from $D$ by a rectifiable curve, then $\delta_{D}\left(E_{0}, E_{1}\right)=\infty$.

(XII) Let $D, E_{0}$, and $E_{1}$ be contained in the closed upper half-plane. Let $\hat{D}$ be the region which is the union of $D$, the reflection of $D$ across the real axis, and the part of $\partial D$ on the real axis. Let $\hat{E}_{0}$ and $\hat{E}_{1}$ have analogous meanings. If $\delta_{\hat{D}}\left(\hat{E}_{0}, \hat{E}_{1}\right)$ is expressed in terms of the extremal length of a family consisting of analytic curves ${ }^{2}$, then

$$
\delta_{\hat{D}}\left(\hat{E}_{0}, \hat{E}_{1}\right)=\frac{1}{2} \delta_{D}\left(E_{0}, E_{1}\right) .
$$

Proof. Let $\delta_{\hat{D}}\left(\hat{E}_{0}, \hat{E}_{1}\right)=\lambda\{\gamma\}$ where $\gamma$ is an analytic curve and let $\delta_{D}\left(E_{0}, E_{1}\right)=\lambda\left\{\gamma^{\prime}\right\}$. Using the notation in (VII), we see immediately that $\left\{\gamma^{\prime}\right\}$ and $\left\{\bar{\gamma}^{\prime}\right\}$ are contained in $\{\gamma\}$. Since $\lambda\left\{\gamma^{\prime}\right\}=\lambda\left\{\bar{\gamma}^{\prime}\right\}$, we find, on applying (V), that $\lambda\{\gamma\} \leqq \lambda\left\{\gamma^{\prime}\right\} / 2$.

In order to prove the inequality in the opposite direction, we first remark that, to define $\lambda\{\gamma\}$, we may restrict $\rho$ to a function symmetric about the real axis. For a curve $\gamma: z=z(t)(0<t<1)$, let $\gamma^{*}$ be

$$
z=\left\{\frac{z(t)}{z(t)}\right.
$$

$$
\begin{aligned}
& \text { if } \Im z(t) \geqq 0 \\
& \text { if } \Im z(t) \leqq 0 .
\end{aligned}
$$

Evidently $\int_{\gamma} \rho d s=\int_{\gamma *} \rho d s$ for a symmetric $\rho$.

Since it is assumed that $\gamma$ is an analytic curve, $\gamma^{*}$ intersects the real axis at only a finite number of points $z_{1}, z_{2}, \cdots, z_{k}$. Let $\Delta_{\nu}$ be the punctured disk $0<\left|z-z_{\nu}\right|<r(\nu=1,2, \cdots, k)$, where $r$ is taken so small that the $\Delta_{\nu}$ are mutually disjoint. The extremal length of the family of curves in $\Delta_{\nu}$ separating $z_{\nu}$ from $\left|z-z_{\nu}\right|=r$ is, by (IX), equal to infinite. Therefore, for arbitrary $\varepsilon>0$ and $\rho$, there exists a closed curve $\gamma_{\nu} \subset \Delta_{\nu}$ encircling $z_{\nu}$ and such that $\int_{\gamma_{\nu}} \rho d s<\varepsilon / k$. On replacing a part of $\gamma^{*} \cap \Delta$, by a part of $\gamma_{\nu}(\nu=1,2, \cdots, k)$, we obtain from $\gamma^{*}$ a

2 This restriction is satisfied in our subsequent applications. It is perhaps superfluous. However, the author has not succeeded in furnishing the proof without it. 
curve $\gamma^{\prime}$ belonging to the family $\left\{\gamma^{\prime}\right\}$ and such that $\int_{\gamma^{\prime}} \rho d s-\varepsilon<\int_{\gamma} \rho d s$. Since $\gamma$ and $\varepsilon$ are arbitrary, we get $\inf _{\gamma^{\prime}} \int_{\gamma^{\prime}} \rho d s \leqq \inf _{\gamma} \int_{\gamma} \rho d s$ for every symmetric $\rho$. Since $\iint_{\hat{D}} \rho^{2} d x d y=2 \iint_{D} \rho^{2} d x d y$, we conclude that $\lambda\left\{\gamma^{\prime}\right\} \leqq$ $2 \lambda\{\gamma\}$.

(XIII) Let $A$ be the annulus $1<|z|<q$ or a region obtained by deleting a finite number of radial slits from this annulus. Let $E_{0}=$ $(|z|=1)$ and $E_{1}=(|z|=q)$. Then $\delta_{A}\left(E_{0}, E_{1}\right)=(\log q) / 2 \pi$, and it is also equal to the extremal length of the family of all radials from $E_{0}$ to $E_{1}$ in $A$.

For the proof, the reader is referred to, e.g., Strebel [20].

\section{Teichmüller's extremal region.}

11. Let $D$ be a doubly connected region and let $\{\gamma\}$ be the family of all closed rectifiable curves in $D$ separating the boundary components. The quantity $2 \pi / \lambda\{\gamma\}$ is called the modulus of $D$ and is denoted by $\bmod D$. As is well known, $D$ can be mapped conformally onto an annulus $1<|z|<q$ where $\log q=\bmod D$.

For $P>0$, the doubly connected region

$$
D_{P}=\{[-1,0] \cup[P, \infty]\}^{c}
$$

where the brackets express a closed interval on the real axis, is called Teichmüller's extremal region. It has the following extremal property (Teichmüller [23]): Let $D$ be a doubly connected region such that one component of $D^{c}$ contains the point $z=0$ as well as a point on $|z|=1$ and the other contains the point $z=\infty$ as well as a point on $|z|=P$. Then $\bmod D \leqq \bmod D_{P}$ and the equality holds if and only if $D$ is a region obtained by rotating $D_{P}$ about the origin.

12. It was proved by Teichmüller [23] that $\Psi(P)=\exp \left(\bmod D_{P}\right)$ is a continuous function of $P$ such that

$$
\lim _{P \rightarrow \infty} \frac{\Psi(P)}{P}=16 .
$$

It is easy to see that

$$
\log \Psi\left(\frac{1}{P}\right)=\frac{\pi^{2}}{\log \Psi(P)} .
$$

On combining (1) and (2), we have

$$
\log \Psi(P) \sim \frac{\pi^{2}}{\log \frac{1}{P}}
$$


13. The following result will be used later:

LemMA 1. Let

$$
\begin{aligned}
& A=(1<|z|<q), \\
& \Gamma=(|z|=1),
\end{aligned}
$$

and

$$
E_{\theta}=\{z ;|z|=q,|\arg z| \leqq \theta\}
$$

Then

$$
\delta_{A}\left(\Gamma, E_{\theta}\right) \sim \frac{1}{\pi} \log \frac{1}{\theta}
$$

for $\theta \rightarrow 0$.

Proof. $\delta_{A}(\Gamma, E)$ is equal to the extremal length $\lambda\{\gamma\}$ where $\{\gamma\}$ is the family of all analytic curves in $A$ connecting $\Gamma$ with $E_{\theta}$ (cf. Wolontis [25]). By (VIII) and (XIII), it is equal to $\delta_{Q}\left(E_{\theta}^{\prime}, E_{\theta}^{\prime \prime}\right) / 4$ where

$$
\begin{aligned}
& Q=(1 / q<|z|<q) \cap(\mathfrak{\Im} z>0), \\
& E_{\theta}^{\prime}=\{z ;|z|=1 / q, 0 \leqq \arg z \leqq \theta\},
\end{aligned}
$$

and

$$
E_{\theta}^{\prime \prime}=\{z ;|z|=q, 0 \leqq \arg z \leqq \theta\} .
$$

Map $Q$ onto the upper half-plane in such a way that $1 / q$ and $q$ correspond to 0 and 1 , respectively. Let $-\alpha$ and $1+\beta(\alpha, \beta>0)$ be the images of $e^{i \theta} / q$ and $q e^{i 3}$, respectively. It is not difficult to see that

$$
\left\{\begin{array}{l}
\alpha \sim c \frac{\theta^{2}}{q} \\
\beta \sim c^{\prime} q \theta^{2}
\end{array}\right.
$$

where $c$ and $c^{\prime}$ are constants independent of $\theta$. The region obtained by deleting the intervals $[-\infty,-\alpha],[0,1]$, and $[1+\beta, \infty]$ from the extended plane is conformally equivalent to Teichmüller's extremal region with

$$
P=\frac{\alpha \beta}{1+\alpha+\beta} \sim c^{\prime \prime} \theta^{4}
$$

Therefore, on applying (VIII) again, we get $\delta_{A}\left(\Gamma, E_{\theta}\right)=\pi /(4 \log \Psi(P))$ and, by (3),

$$
\delta_{A}\left(\Gamma, E_{\theta}\right) \sim \frac{1}{4 \pi} \log \frac{1}{P} \sim \frac{1}{\pi} \log \frac{1}{\theta} \quad \text { for } \theta \rightarrow 0 .
$$




\section{Koebe's distortion theorem.}

14. The following is a slight modification of the original form of Koebe's well-known distortion theorem, which will be used frequently:

Let $\phi(z)$ be a function which is univalent and regular in $|z|<\varepsilon_{0}$ with $\varphi(0)=0$ and $\varphi^{\prime}(0)=1$. Then there are numbers $a(\varepsilon)$ and $b(\varepsilon)$ which are independent of $\rho$ and have the properties that

$$
a(\varepsilon) \leqq|\mathcal{P}(z)| \leqq b(\varepsilon) \quad \text { on }|z|=\varepsilon<\varepsilon_{0}
$$

and

$$
\lim _{\varepsilon \rightarrow 0} \frac{a(\varepsilon)}{\varepsilon}=\lim _{\varepsilon \rightarrow 0} \frac{b(\varepsilon)}{\varepsilon}=1
$$

In fact, we may take $a(\varepsilon)=\varepsilon \varepsilon_{0}^{2} /\left(\varepsilon+\varepsilon_{0}\right)^{2}$ and $b(\varepsilon)=\varepsilon \varepsilon_{0}^{2} /\left(\varepsilon-\varepsilon_{0}\right)^{2}$.

\section{Quasi-conformal mappings.}

15. In Chapters IV and V, we shall make use of quasi-conformal mappings to illustrate our results by examples. As in the type problem of Riemann surfaces, they are utilized to replace a given region by a simpler one.

A sense-preserving topological mapping $w=T(z)$ of a region $D$ onto another is said to be quasi-conformal if there exists a finite number $K$ such that $\bmod T(Q) \leqq K \bmod Q$ for any quadrilateral $Q \subset D$ (Ahlfors [1]). Here, $\bmod Q$ of a quadrilateral $Q$ means the extremal distance between two opposite sides of $Q$. The minimum value of $K$ is called the maximal dilatation of $T$.

For the proofs of the following properties (I)-(III), the reader is referred to Ahlfors [1]:

(I) If $T$ is quasi-conformal of maximal dilatation $K$, then $\bmod T(A) \leqq K \bmod A$ for any doubly connected region $A \subset D$.

(II) Let $E$ be a set which is contained in a finite number of analytic arcs. Let $D$ be a region containing $E$, and let $T$ be a topological mapping of $D$ which is quasi-conformal in $D-E$. Then it is quasiconformal in $D$ with the same maximal dilatation.

(III) If $T$ is a topological mapping of class $C^{1}$, then the maximal dilatation is given by $K=\sup _{z \in D}\left(\left|T_{z}\right|+\left|T_{\bar{z}}\right|\right) /\left(\left|T_{z}\right|-\left|T_{\bar{z}}\right|\right)$ where $T_{z}$ and $T_{\bar{z}}$ are complex derivatives.

(IV) Let $\{\gamma\}$ be a family of curves in $D$. Let $T$ be a quasiconformal mapping of class $C^{1}$ with the maximal dilatation $K$. Then

$$
\lambda\{T(\gamma)\} \leqq K \lambda\{\gamma\}
$$

The proof is found in Hersch [9] ${ }^{1}$. 
Remark. Even if $T$ is not of class $C^{1}$ throughout $D$, this inequality holds under, e.g., the following restriction: $T$ is of $C^{1}$ in $D$ except for a countable number of analytic arcs clustering nowhere in $D$, i.e., every point of $D$ has a neighborhood intersecting at most a finite number of the arcs, and every $\gamma$ is the union of a countable number of analytic arcs clustering nowhere in $D$. This generalization will be needed in No. 35 .

\section{Circular and Radial Slit Disks}

\section{Circular slit disks.}

16. Let $D$ be a plane region containing the point $z=0$, and let $\Gamma$ be a boundary component. The problem of minimizing $M(f)$ in $\mathfrak{F}_{F}$ for a region of finite connectivity has been discussed by Rengel [14]. To consider it for a region of arbitrary connectivity, in particular to show the uniqueness of the minimizing function, Sario [16] introduced the functional

$$
J(f)=\int_{\partial D} \log |f| \cdot d \arg f \quad\left(f \in \mathfrak{F}_{\Gamma}\right) .
$$

Here the line integral means $\lim _{n \rightarrow \infty} \int_{\partial D_{n}} \log |f| \cdot d \arg f$ for an exhaustion $D_{n} \uparrow D$; the limiting value exists and is independent of the exhaustion. He proved the existence of a function $g_{0}$ such that

$$
M\left(g_{0}\right)=m\left(g_{0}\right)
$$

and

$$
2 \pi \log M\left(g_{0}\right)=J(f)-D\left(\log |f|-\log \left|g_{0}\right|\right)
$$

for all $f \in \mathfrak{F}_{r}$, where the second term means the Dirichlet integral over $D$. Evidently $g_{0}$ is the unique function which minimizes $J(f)$.

From these relations we can derive the following facts (Sario [16]):

( I ) There exists a function $g_{0} \in \mathfrak{F}_{F}$ such that $M\left(g_{0}\right)=\min _{f \in \mathfrak{F}_{\Gamma}} M(f)=$ $r(\Gamma)$. If $r(\Gamma)<\infty$, the minimizing function is determined uniquely. It maps $D$ onto a circular slit disk $|w|<r(\Gamma)$, where the area of slits, i.e., $g_{0}(\partial D-\Gamma)^{*}$, vanishes,

(II) Let $0 \in D_{n} \uparrow D$ be an exhaustion and let $\Gamma_{n}$ be the component of $\partial D_{n}$ separating $D_{n}$ from $\Gamma$. Then

$$
r(\Gamma)=\lim _{n \rightarrow \infty} r\left(\Gamma_{n}\right) .
$$

If $r(\Gamma)<\infty$, the sequence $\left\{g_{n}\right\}$ of the minimizing functions on $D_{n}$ converges to $g_{0}$ uniformly on each compact set in $D$. 
17. By making use of this result, we can express $r(\Gamma)$ in terms of extremal length. Let $\varepsilon_{0}$ be a small number such that $|z| \leqq \varepsilon_{0}$ is contained in $D$. For $0<\varepsilon<\varepsilon_{0}$, the numbers $a(\varepsilon)$ and $b(\varepsilon)$ were defined in No. 14. The following theorem has been proved, in essence, by Jurchescu [10]:

THeorem 2. Let $\{\gamma\}_{\varepsilon}$ be the family of all closed curves in $D_{\varepsilon}=$ $D-(|z| \leqq \varepsilon)$ which separate $\Gamma$ from the point $z=0$. Then

$$
\log \frac{r(\Gamma)}{b(\varepsilon)} \leqq \frac{2 \pi}{\lambda\{\gamma\}_{\varepsilon}} \leqq \log \frac{r(\Gamma)}{a(\varepsilon)}
$$

and, therefore,

$$
\log r(\Gamma)=\lim _{\varepsilon \rightarrow 0}\left(\log \varepsilon+\frac{2 \pi}{\lambda\{\gamma\}_{\varepsilon}}\right)
$$

The result remains valid if the $\gamma$ are restricted to analytic curves.

Proof. Consider the metric given by $\rho=\left|g_{0}^{\prime}\right| /\left|g_{0}\right|$. Since the area of the circular slits is zero, $\iint_{D_{\varepsilon}} \rho^{2} d x d y \leqq 2 \pi \log \left(r\left(\Gamma^{\top}\right) / a(\varepsilon)\right)$. Therefore,

$$
\lambda\{\gamma\}_{\varepsilon} \geqq(2 \pi)^{2} / 2 \pi \log (r(\Gamma) / a(\varepsilon)) .
$$

To prove the left inequality, take an exhaustion $D_{n} \uparrow D$ and consider the family $\left\{\gamma_{n}\right\}_{\varepsilon}$ of all closed curves $\gamma_{n}$ in $D_{n}-(|z| \leqq \varepsilon)$ separating $\Gamma_{n}$ from $z=0$. Since $D_{n}$ is of finite connectivity, the proposition (IX), No. 9, shows that $2 \pi / \lambda\left\{\gamma_{n}\right\}_{\varepsilon} \geqq \log \left(r\left(\Gamma_{n}\right) / b(\varepsilon)\right)$. When we take the limit for $n \rightarrow \infty$, we have by virtue of the relation $\lambda\{\gamma\}_{\varepsilon} \leqq \lambda\left\{\gamma_{n}\right\}_{\varepsilon}$ that

$$
2 \pi / \lambda\{\gamma\}_{\varepsilon} \geqq \log (r(\Gamma) / b(\varepsilon)) .
$$

18. The following criterion for weakness due to Grötzsch [7] will be useful in the next chapter:

THEOREM 3. In order that $\Gamma$ be weak, it is necessary and sufficient that, for an arbitrary positive number $l$, there exist a finite number of doubly connected regions $A_{1}, A_{2}, \cdots A_{k}$ in $D-(|z| \leqq \varepsilon)$ satisfying the following conditions:

(i) The $A_{\text {, }}$ are mutually disjoint,

(ii) $A$, separates $\Gamma$ from $(|z| \leqq \varepsilon)(\nu=1,2, \cdots, k)$ and separates $A_{-1}$ from $A_{*_{+1}}(\nu=2,3, \cdots, k-1)$,

$$
\sum_{\nu=1}^{k} \bmod A_{\nu} \geqq l .
$$


Proof. Sufficiency: By (V), No. 9, and by Theorem 2, $l \leqq$ $\sum_{\nu=1}^{k} \bmod A_{\nu} \leqq 2 \pi / \lambda\{\gamma\}_{\varepsilon} \leqq \log (r(\Gamma) /(\varepsilon))$. Therefore, $r(\Gamma)=\infty$ and, by Theorem $1, \Gamma$ is weak.

Necessity: Take an exhaustion $(|z| \leqq \varepsilon) \subset D_{1} \subset D_{2} \subset \cdots \subset D_{n} \subset$ $\cdots \uparrow D$ and consider the extremal function $\mathrm{g}_{n}$ on $D_{n}$. By Koebe's distortion theorem, No. 14, the image of $|z|=\varepsilon$ is contained in $a(\varepsilon) \leqq|w| \leqq b(\varepsilon)$, so that the set $b(\varepsilon)<|w|<r\left(\Gamma_{n}\right)$ minus the circular slits is contained in the image of $D_{n}-(|z| \leqq \varepsilon)$. From the annulus $b(\varepsilon)<|w|<r\left(\Gamma_{n}\right)$, delete all the concentric circles containing the circular slits. Then we get a finite number of concentric annuli $A_{1}^{\prime}, A_{2}^{\prime}, \cdots, A_{k}^{\prime}$ such that $\sum_{\nu=1}^{k} \bmod A_{\nu}^{\prime}=\log \left(r\left(\Gamma_{n}\right) / b(\varepsilon)\right)$. Since $r(\Gamma)=\lim _{n \rightarrow \infty} r\left(\Gamma_{n}\right)=\infty$, we can take $n$ so large that the right hand side is greater than the given $l$. The inverse images $A_{1}, A_{2}, \cdots, A_{k}$ of $A_{1}^{\prime}, A_{2}^{\prime}, \cdots, A_{k}^{\prime}$ are what we desired.

REMARK. We see from this theorem that the weakness of $\Gamma$ depends merely on the configuration of $\partial D$ near $l$. Furthermore, by (I), No. 15, the weakness is invariant under quasi-conformal mappings.

\section{Radial slit disks for special regions.}

19. Unlike the case of the functional $M(f)$, the function maximizing $m(f)$ does not exist in general; by slightly modifying the example given by Strebel [20], we get a region on which $m(f)<R(\Gamma)=\sup _{f \in \mho_{r}}$ $m(f)$ for all $f \in \mathfrak{F}_{\Gamma}$.

Under a restriction, however, we get a result analogous to that of No. 15. Let $G$ be a region containing the point $z=0$ and such that a component $\Gamma$ of $\partial G$ consists of a closed analytic curve which is isolated, i.e., $\overline{\partial G-\Gamma} \cap \Gamma=\phi$. Let $\mathfrak{U}_{\Gamma}$ be the subfamily of $\widetilde{\mho}_{\Gamma}$ consisting of all functions with $M(f)=m(f)$. On this family Sario [17, 18] introduced the functional

$$
l(f)=2 \pi \log m(f)-\int_{\partial D-r} \log |f| \cdot d \arg f
$$

and proved the existence of a function $f_{0} \in \mathfrak{A}_{\Gamma}$ such that

$$
2 \pi \log m\left(f_{0}\right)=I(f)+D\left(\log |f|-\log \left|f_{0}\right|\right)
$$

for all $f \in \mathfrak{A}_{\Gamma}$. Evidently $f_{0}$ is the unique maximizing function of $I(f)$ in $\mathfrak{U}_{\Gamma}$.

We can derive from this relation the following facts (Sario [18]), which have been obtained by Rengel [14] for a region $G$ of finite connectivity: 
(I) $R(\Gamma)$ is finite. $f_{0}$ is the unique function maximizing $m(f)$ in $\mathfrak{A}_{\Gamma}$. It maps $G$ onto a radial slit disc $|w|<R(\Gamma)$, where the area of slits, i.e., $f_{0}(\partial G-\Gamma)^{*}$, vanishes.

(II) Let $\left\{G_{n}\right\}$ be a sequence of regions such that $0 \in G_{n} \uparrow G$ and $\partial G_{n}$ consists of $\Gamma$ and a finite number of closed analytic curves. Then

$$
R(\Gamma ; G)=\lim _{n \rightarrow \infty} R\left(\Gamma_{n} ; G_{n}\right)
$$

and the sequence $\left\{f_{n}\right\}$ of the maximizing functions on $G_{n}$ converges to $f_{0}$ uniformly on each compact set in $G \cup I^{\prime}$.

20. Let $\{\gamma\}_{\varepsilon}$ be the family of rectifiable curves which connect $|z|=\varepsilon$ with $I^{\prime}$ in $G-(|z| \leqq \varepsilon)$. In a method similar to the proof of Theorem 2 we can obtain the following relations:

$$
\frac{\left(\log \frac{R(\Gamma)}{b(\varepsilon)}\right)^{2}}{\log \frac{R(\Gamma)}{a(\varepsilon)}} \leqq 2 \pi \lambda\{\gamma\}_{\varepsilon} \leqq \log \frac{R(\Gamma)}{a(\varepsilon)},
$$$$
\log R(\Gamma)=\lim _{\varepsilon \rightarrow 0}\left(\log \varepsilon+2 \pi \lambda\{\gamma\}_{\varepsilon}\right) \text {. }
$$

Here $\{\gamma\}_{\varepsilon}$ can be replaced by the subfamily of analytic curves.

10. Characterizations of $R(\Gamma)$.

21. Let $D$ be an arbitrary region containing the point $z=0$. Let $\left\{\Omega_{n}\right\}_{n=1}^{\infty}$ be a defining sequence of $\Gamma$ such that $0 \notin \Omega_{n}(n=1,2, \cdots)$. Then $G_{n}=D-\Omega_{n}$ is a region and its boundary component $\Gamma_{n}-\partial G_{n} \cap \partial \Omega_{n}$ satisfies the condition of No. 19 .

Theorem 4. $\left\{R\left(\Gamma_{n}, G_{n}\right)\right\}_{n=1}^{\infty}$ is an increasing sequence and $R(\Gamma)=$ $\lim _{n \rightarrow \infty} R\left(\Gamma_{n} ; G_{n}\right)$.

Proof. $\left\{R\left(\Gamma_{n} ; G_{n}\right)\right\}$ is an increasing sequence by (6).

For an arbitrary $\varepsilon>0$, there exists an $f(z) \in \mathfrak{F}_{r}$ such that $m(f)>$ $R(\Gamma)-\varepsilon / 2$. Then there exists an $n_{0}$ such that the $m$ of this $f(z)$ on $G_{n}$ (we denote it by $m_{n}(f)$ ) has the property that $m_{n}(f)>m(f)-\varepsilon / 2$ whenever $n \geqq n_{0}$. Therefore, $\quad R\left(\Gamma_{n} ; \quad G_{n}\right) \geqq m_{n}(f)>R(\Gamma)-\varepsilon$ and $\underline{\lim }_{n \rightarrow \infty} R\left(\Gamma_{n} ; G_{n}\right) \geqq R(\Gamma)$.

Next, let $A_{n}$ be the doubly connected region bounded by $\Gamma_{n}$ and $\Gamma$. Then $\Gamma$ is an isolated boundary component of the region $\widetilde{G_{n}}=G_{n} \cup A_{n} \cup \Gamma_{n}$. $\Gamma$ is not necessarily a closed analytic curve, but from the result of No. 19 we can see the existence of the function $\tilde{f}_{n}(z)$ in $\widetilde{F}_{F}$ of $\tilde{G}_{n}$ such that $m\left(\tilde{f}_{n}\right)=R\left(\Gamma ; \widetilde{G}_{n}\right)$. Evidently $\tilde{f_{n}}(z)$ belongs to $\widetilde{F}_{F}$ of $D$. By $(6)$, 
$R\left(\Gamma_{n} ; G_{n}\right) \leqq R\left(\Gamma ; \tilde{G}_{n}\right) . \quad$ Consequently, $R\left(\Gamma_{n} ; G_{n}\right) \leqq R\left(\Gamma ; \tilde{G}_{n}\right)=m\left(\tilde{f}_{n}\right) \leqq$ $R(\Gamma)$ and $\varlimsup_{n \rightarrow \infty} R\left(\Gamma ; G_{n}\right) \leqq R(\Gamma)$.

This reasoning remains valid for the case where $R(\Gamma)=\infty$.

REMARK. Combining Theorem 4 with Theorem 1 , we see that $\lim _{n \rightarrow \infty} R\left(\Gamma_{n} ; G_{n}\right)<\infty$ implies the strength of $\Gamma$. This fact was proved by Sario [17].

22. Let $\{\gamma\}_{\varepsilon}$ be the family of curves $\gamma: z=z(t)(0<t<1)$ in $D-(|z| \leqq \varepsilon)$ such that $\bigcap_{\varepsilon>0} \overline{\{z(t) ; 0<t<\varepsilon\}} \subset(|z|=\varepsilon)$ and $\bigcap_{\varepsilon>0}$ $\overline{\{z(t) ; 1-\varepsilon<t<1\}} \subset \Gamma$. Let $\left\{\gamma_{n}\right\}_{\varepsilon}$ be the corresponding family in $G_{n}$. Strebel [22] has proved the relation $\lambda\{\gamma\}_{\varepsilon}=\lim _{n \rightarrow \infty} \lambda\left\{\gamma_{n}\right\}_{\varepsilon}$. On combining this with (5), (6), and Theorem 4, we have

THEOREM 5.

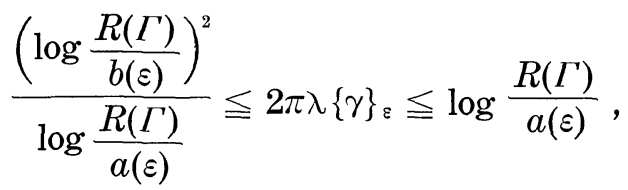

$$
\begin{aligned}
& \log R(\Gamma)=\lim _{\varepsilon \rightarrow 0}\left(\log \varepsilon+2 \pi \lambda\{\gamma\}_{\varepsilon}\right) .
\end{aligned}
$$

Here $\gamma$ can be restricted to the curve which is the union of a countable number of analytic arcs which cluster nowhere in $D$ (cf. No. 15, Remark).

REMARK. The exponential of the right hand side of the second relation was called "extremal Durchmesser" by Strebel [22]. On combining Theorem 5 with Theorem 1, or directly from (XI), No. 10, we see that $\lambda\{\gamma\}_{\varepsilon}<\infty$ implies the strength of $\Gamma$. This result was generalized for open Riemann surfaces by Constantinescu [4].

23. For an exhaustion $D_{n} \uparrow D$ in the ordinary sense, it has not been proved whether $\lim _{n \rightarrow \infty} R\left(I_{n} ; D_{n}\right)$ exists or not. We obtain merely the following

THeOREM 6. Let $\Delta$ be a region such that $0 \in \Delta, \bar{\Delta} \subset D$, and bounded by a finite number of closed analytic curves. Denote by $\Gamma_{4}$ the component of $\partial \Delta$ which separates $\Delta$ from $\Gamma$. Then

$$
R(\Gamma)=\lim _{\Delta \rightarrow D} R\left(\Gamma_{\Delta} ; \Delta\right),
$$

where the right hand side is a directed limit.

Proof. For $\varepsilon>0$, there exists by Theorem 4 an $n$ such that 
$R(\Gamma)-\varepsilon<R\left(\Gamma_{n} ; G_{n}\right)$. By Theorem $5 R\left(\Gamma_{n} ; G_{n}\right) \leqq R\left(\Gamma_{\Delta} ; \Delta\right)$ for any $\Delta \supset \Gamma_{n} \cup\{0\}$. Therefore, $R(\Gamma) \leqq \lim _{\Delta \rightarrow D} R\left(\Gamma_{\Delta} ; \Delta\right)$. On the other hand, for $\varepsilon>0$ and a compact set $K \subset \overline{\subset D}$, take an $n_{0}$ such that $K \subset G_{n_{0}}$. There exists, by (II), No. 19, a $\Delta \subset G_{n_{0}}$ such that $R\left(\Gamma_{4} ; \Lambda\right) \subset R\left(\Gamma_{n_{0}} ; G_{n_{0}}\right)+\varepsilon$, and, therefore, $R\left(\Gamma_{\Delta} ; \Delta\right)<R(\Gamma)+\varepsilon$. Consequently $\lim _{\Delta \rightarrow D} R\left(\Gamma_{\Delta} ; \Delta\right) \leqq R(\Gamma)$.

REMarK. On combining Theorem 6 with Theorem 1 we see that $\underline{\lim }_{\Delta \rightarrow D} R\left(\Gamma_{4} ; \Delta\right)<\infty$ implies the strength of $\Gamma$. Sario [18] has shown that $\Gamma$ is strong if $\varlimsup_{4 \rightarrow D} R\left(\Gamma_{\Delta} ; \Delta\right)<\infty$.

\section{Unsolved problems.}

24. As we pointed out in No. 7, the following problem has not been solved:

(1) Is $R(\Gamma)<\infty$ necessary for the strength of $\Gamma$ ?

Since the maximizing function of $m(f)$ in $\mathfrak{F}_{F}$, or equivalently the minimizing function of diam $\varphi(\Gamma)$ in $\mathfrak{B}$, does not exist in general, the case is different from that of a weak boundary component. The example of Strebel [20] stated in No. 19 is for $R(\Gamma)>\infty$, and it does not answer this question.

Let $\left\{G_{n}\right\}_{n=1}^{\infty}$ be the sequence introduced in No. 21 and let $f_{n}(z)$ be the extremal function on $G_{n}$. Since $\left\{f_{n}\right\}_{n=1}^{\infty}$ is a normal family, we may assume that $f_{n}$ converges to a univalent function $f(z)$. One can imagine that, if $R(\Gamma)=\infty$, then $f(\Gamma)$ would be a point. However, we can only prove that $f(\Gamma)$ consists of the point $w=\infty$ and possibly of radial segments emanating from it whose arguments form a set of measure zero (Strebel [22]). Such line segments appear in our Example 10, Nos. 39,40 . Nevertheless the boundary component of this example is unstable, because we can map it onto a region such that $f(\Gamma)$ is a point and $f(\partial D-\Gamma)$ consists of circles (No. 39).

We have several other unsolved problems as follows:

(2) Is strength a boundary property?

(3) Is $\varlimsup_{4 \rightarrow D} R\left(\Gamma_{\Delta} ; \Delta\right)$ equal to $\underline{\lim }_{\Delta \rightarrow D} R\left(\Gamma_{\Delta} ; \Delta\right)$ ?

(4) Is strength preserved under quasi-conformal mappings?

\section{CRITERIA FOR WEAKNESS AND INSTABILJTY}

In this chapter we consider Problem A presented in No. 4. Several sufficient conditions for weakness have been obtained by Savage [19]. Here we shall consider some special regions and attempt to get more concrete necessary or sufficient conditions. 
12. Boundary on the positive real axis.

25. Let $\left\{a_{n}\right\}_{n=1}^{\infty}$ and $\left\{b_{n}\right\}_{n=0}^{\infty}$ be sequences of positive numbers such that

$$
\begin{aligned}
1<b_{n-1} & \leqq a_{n}<b_{n} \quad(n=1,2, \cdots), \\
\lim _{n \rightarrow \infty} a_{n} & =\infty .
\end{aligned}
$$

Denote by $[a, b]$ the closed interval on the real axis. Then

$$
D=(|z|<\infty)-\bigcup_{n=1}^{\infty}\left[b_{n-1}, a_{n}\right]
$$

is a region and $\Gamma=\{\infty\}$ is its boundary component. The present section is devoted to discussing the following problem: When is $\Gamma$ weak and when is it unstable?

26. THEOREM 7. (i) $I f$

$$
\sum_{n=1}^{\infty}\left(\frac{b_{n}}{a_{n}}-1\right)=\infty
$$

then $\Gamma$ is weak.

(ii) If

$$
\lim _{n \rightarrow \infty} \frac{b_{n}}{a_{n}}=1^{3}
$$

and

$$
\sum_{n=1}^{\infty} \frac{1}{\log \frac{1}{\left(b_{n} / a_{n}\right)-1}}<\infty
$$

then $\Gamma$ is unstable.

Proof. (i) Consider the annuli $A_{n}=\left(a_{n}<|z|<b_{n}\right)(n=1,2, \cdots)$. Since $\sum \bmod A_{n}=\sum \log \left(b_{n} / a_{n}\right)=\infty$, Theorem 3 shows that $\Gamma$ is weak.

(ii) Let $A_{1}, A_{2}, \cdots, A_{k}$ be doubly connected regions satisfying the conditions (i) and (ii) of Theorem 3. For any $A_{\nu}$, there exists an $n$ such that $A_{\nu}$ passes through the open interval $\left(a_{n}, b_{n}\right)$ and a component of $A_{\nu}$ contains 0 as well as $a_{n}$. The region

$$
D^{(n)}=\left\{\left[0, a_{n}\right] \cup\left[b_{n}, \infty\right]\right\}^{c}
$$

is conformally equivalent to Teichmüller's extremal region with $P=$ $\left(b_{n} / a_{n}\right)-1$. By the extremal property of $D^{(n)}$, No. 11, the sum of the ${ }^{3}$ If $\overline{\lim }_{n \rightarrow \infty} b_{n} / a_{n}>1$, then $\Gamma$ is weak by (i), Theorem 7 
moduli of all such $A_{\nu}$ does not exceed $\bmod D^{(n)}=\log \Psi\left(\left(b_{n} / a_{n}\right)-1\right)$.

$$
\sum_{\nu=1}^{k} \bmod A_{\nu} \leqq \sum_{n=1}^{\infty} \log \Psi\left(\frac{b_{n}}{a_{n}}-1\right) \text {. }
$$

By (3), No. 12,

$$
\log \Psi\left(\frac{b_{n}}{a_{n}}-1\right) \sim \frac{\pi^{2}}{\log \frac{1}{\left(b_{n} / a_{n}\right)-1}} .
$$

Therefore, the right hand side of (10) converges and, by Theorem $3, \Gamma$ is unstable.

ExAmple 1. $a_{n}=2 n+1, b_{n}=2 n+2$. Evidently (7) diverges so that $\Gamma$ is weak.

EXAMPLE 2. $a_{n}=n^{k}, b_{n}=n^{k}+1(k>1)$. Since (7) converges and (9) diverges, we cannot decide by Theorem 7 (see also No. 27).

ExAMPle 3. $a_{n}=e^{n}, b_{n}=e^{n}+1$. Similarly, we cannot decide (see also No. 27).

ExAMPLE 4. $a_{n}=e^{n^{\alpha}}, b_{n}=e^{n^{\alpha}}+1(\alpha>1) . \quad \Gamma$ is unstable by (ii).

27. We derive another criterion applicable to Examples 2 and 3 . To this end, we first prove

LeMMA 2. For the doubly connected region

$$
A_{h}=(1<|z|<q)-[1+h, q)
$$

where $h>0$ and $q$ is fixed,

$$
\bmod A_{h} \sim \frac{\pi^{2}}{2 \log \frac{1}{h}}
$$

Proof. By (VIII), No. 9, $\bmod A_{h}=4 \pi / \lambda\{\gamma\}$ where $\{\gamma\}$ is the family of rectifiable curves in $Q=A_{l} \cap(\mathfrak{I} z>0)$ joining $[-q,-1]$ with $[1,1+h]$. Map $Q$ conformally onto the upper half-plane in such a manner that $-q,-1,1$ correspond to $-\infty,-1,0$, respectively. The image $P$ of $1+h$ has the property that

$$
P \sim c h^{2}
$$

for $h \rightarrow 0$

where $c$ is a constant independent of $h$. From (VIII), No. 9, we conclude that 


$$
\bmod A_{h}=\log \Psi(P) \sim \frac{\pi^{2}}{\log \frac{1}{P}} \sim \frac{\pi^{2}}{2 \log \frac{1}{h}} \quad(h \rightarrow 0)
$$

THEOREM 8. Suppose that $\lim _{n \rightarrow \infty} b_{n} / a_{n}=1$. If $a_{n+1} / a_{n}$ is bounded away from 1, then $\Gamma$ is weak if and only if

$$
\sum_{n=1}^{\infty} \frac{1}{\log \frac{1}{\left(b_{n} / a_{n}\right)-1}}=\infty
$$

Proof. If the series converges, $\Gamma$ is unstable by (ii) of Theorem 7 . Conversely, suppose that the series diverges. The doubly connected region $A_{n}=\left(a_{n}<|z|<a_{n+1}\right)-\left[b_{n}, a_{n+1}\right)$ is conformally equivalent to the region $A_{n}^{\prime}=\left(1<|z|<a_{n+1} / a_{n}\right)-\left[b_{n} / a_{n}, a_{n+1} / a_{n}\right)$. By the assumption $1<1+\delta<a_{n+1} / a_{n}$ and, therefore, $A_{n}^{\prime \prime}=(1<|z|<1+\delta)-\left[b_{n} \mid a_{n}, 1+\delta\right) \subset A_{n}^{\prime}$ so that $\bmod A_{n}^{\prime \prime} \leqq \bmod A_{n}$. By Lemma 2

$$
\bmod A_{n}^{\prime \prime} \sim \frac{\pi^{2}}{2 \log \frac{1}{\left(b_{n} / a_{n}\right)-1}} \quad(n \rightarrow \infty) .
$$

Consequently, the assumption implies that $\sum \bmod A_{n}=\infty$, and we infer from Theorem 3 that $\Gamma$ is weak.

EXAMPle 3 (No. 26). $a_{n}=e^{n}, b_{n}=e^{n}+1$. By Theorem 8, $\Gamma$ is weak.

ExAmPle 2 (No. 26). $a_{n}=n^{k}, b_{n}=n^{k}+1(k>1)$. Since $a_{n+1} / a_{n}=$ $(n+1)^{k} / n^{k}$ is not bounded away from 1 , the above theorem is not applicable. However, we can see as follows that $\Gamma$ is weak. For simplicity, we consider the case $k=2$; the general case can be treated in a similar fashion. Consider the region $A_{n}=\left(a_{2^{n}}<|z|<a_{2^{n+1}}\right)-$ $\left[b_{2^{n}}, a_{2^{n+1}}\right)$, which is conformally equivalent to $(1<|z|<4)-\left[1+2^{-2 n}, 4\right)$. By Lemma $2, \bmod A_{n} \sim \pi^{2} /(4 n \log 2)$ for $n \leftarrow \infty$ and $\sum \bmod A_{n}=\infty$. It follows from Theorem 3 that $\Gamma$ is weak.

More generally, this result can be stated as follows:

THeOREM 8'. Suppose that $\lim _{n \rightarrow \infty} b_{n} / a_{n}=1$ and that there exists a subsequence $\left\{n_{i}\right\} \subset\{n\}$ such that $a_{n_{i+1}} \mid a_{n_{i}}$ is bounded away from 1 and

$$
\sum_{i=1}^{\infty} \frac{1}{\log \frac{1}{\left(b_{n_{i}} / a_{n_{i}}\right)-1}}=\infty
$$

Then $\Gamma$ is weak. 
28. When $a_{n+1} / a_{n}$ is not bounded away from 1, we may also apply the following criterion:

Theorem 9. Suppose $\lim _{n \rightarrow \infty} b_{n} / a_{n}=1$ and $\lim _{n \rightarrow \infty} a_{n+1} / a_{n}=1 . \quad$ If

$$
\lim _{n \rightarrow \infty} \frac{\log \left(b_{n} / a_{n}\right)}{\log \left(a_{n+1} / a_{n}\right)}
$$

exists, then

$$
\sum_{n=1}^{\infty} \frac{\log \left(a_{n+1} / a_{n}\right)}{\log \frac{1}{\left(\frac{b_{n}}{a_{n}}\right)^{1 / \log \left(a_{n+1} / a_{n}\right)}-1}}=\infty
$$

implies that $\Gamma$ is weak.

Proof. Consider the doubly connected region $A_{n}^{\prime}=\left(1<|z|<q_{n}\right)-$ $\left[1+h_{n}, q_{n}\right) \quad(n=1,2, \cdots)$, where $0<h_{n}<q_{n}-1$ and $\lim _{n \rightarrow \infty} q_{n}=1$. Map the annulus $1<|z|<q_{n}$ onto $1<|w|<e$ by the quasi-conformal mapping

$$
w=T_{n}(z)=r^{1 / \log q_{n} e^{i \theta}} \quad\left(z=r e^{i \vartheta}\right) .
$$

Its dilatation equals $1 / \log q_{n}$ provided $n$ is so large that $q_{n}<e$. The

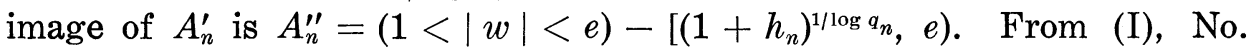
15 , we have

$$
\log q_{n} \cdot \bmod A_{n}^{\prime \prime} \leqq \bmod A_{n}^{\prime} .
$$

Now suppose that $\lim _{n \rightarrow \infty}\left(\log \left(1+h_{n}\right)\right) / \log q_{n}$ exists. If

$$
\lim _{n \rightarrow \infty}\left(1+h_{n}\right)^{1 / \log q_{n}}>1,
$$

then $\bmod A_{n}^{\prime \prime}$ and $\log \left\{1 /\left[\left(1+h_{n}\right)^{1 / \log q_{n}}-1\right]\right\}$ are bounded and bounded away from zero. Hence the divergence of

$$
\sum_{n=1}^{\infty} \frac{\log q_{n}}{\log \frac{1}{\left(1+h_{n}\right)^{1 / \log q_{n}}-1}}
$$

implies that $\sum_{n=1}^{\infty} \log q_{n} \cdot \bmod A_{n}^{\prime \prime}=\infty$ and, by (14), that $\sum_{n=1}^{\infty} \bmod A_{n}^{\prime}=\infty$. If $\lim _{n \rightarrow \infty}\left(1+h_{n}\right)^{1 / \log q_{n}}=1$ we obtain by Lemma 2

$$
\begin{aligned}
& \log A_{n}^{\prime \prime} \sim \frac{\pi^{2}}{2 \log \frac{1}{\left(1+h_{n}\right)^{1 / \log q_{n}-1}}} \\
& (n \rightarrow \infty) \text {. }
\end{aligned}
$$

Therefore, the divergence of (16) again implies that of $\sum_{n=1}^{\infty} \bmod A_{n}^{\prime}$. 
In the given region, consider $A_{n}=\left(a_{n}<|z|<a_{n+1}\right)-\left[b_{n}, a_{n+1}\right)$. It is conformally equivalent to the above $A_{n}^{\prime}$ for $1+h_{n}=b_{n} / a_{n}$ and $q_{n}=$ $a_{n+1} / a_{n}$. Therefore, $\sum_{n=1}^{\infty} \bmod A_{n}=\infty$ and $\Gamma$ is weak.

This criterion is applicable to Example 2.

EXAMPLE 5. $a_{n}=n, b_{n}=n+e^{-n}$. In this case (7) converges and (9) diverges, so that we cannot decide by Theorem 7. Since $a_{n+1} / a_{n}$ is not bounded away from zero, we cannot apply Theorem $8 .^{4}$ For every subsequence such that $\lim _{i \rightarrow \infty} a_{n_{i+1}} \mid a_{n_{i}}>1$, (12) converges, and we cannot use Theorem $8^{\prime}$. (14) also converges and, therefore 9 is inapplicable. We have not been able to decide whether $\Gamma$ is weak or unstable. In general, for $a_{n}=n, b_{n}=n+e^{-n^{\alpha}}(\alpha>0), \Gamma$ is unstable for $\alpha>1$ but it is unknown if it remains true for $0<\alpha \leqq 1$.

\section{A generalization.}

29. Consider the case where the intervals are distributed on the whole real axis. We treat again the simplest case.

PROBLEM. Let $\left\{a_{n}\right\}_{n=1}^{\infty}$ and $\left\{b_{n}\right\}_{n=0}^{\infty}$ be the sequence of positive numbers such that

$$
\begin{gathered}
0<b_{n-1} \leqq a_{n}<b_{n} \quad(n=1,2, \cdots) \\
\lim _{n \rightarrow \infty} a_{n}=\infty .
\end{gathered}
$$

Consider the region

$$
\tilde{D}=(|z|<\infty)-\bigcup_{n=1}^{\infty}\left[b_{n-1}, a_{n}\right]-\bigcup_{n=1}^{\infty}\left[-a_{n},-b_{n-1}\right] .
$$

Under what condition is $\tilde{\Gamma}=\{\infty\}$ a weak boundary component of $\tilde{D}$ ?

This problem can be reduced to the case which we discussed in the previous section. More precisely, let $\Gamma=\{\infty\}$ be a boundary component of

$$
D=(|z|<\infty)-\bigcup_{n=1}^{\infty}\left[b_{n-1}, a_{n}\right]
$$

then we have

THEOREM 10. $\tilde{\Gamma}$ is weak if and only if $\Gamma$ weak.

Proof. If $\Gamma$ is unstable, then, since $\tilde{D} \subset D, \tilde{\Gamma}$ is unstable by the definition.

${ }_{4}$ The author is indebted to Professor R. Redheffer for the argument that follows in this example. 
Suppose that $\tilde{\Gamma}$ is unstable. Since weakness is a boundary property (No. 18), we may assume without loss of generality that $b_{0}>1$. By Theorem $2, \lambda\{\gamma\}>0$ where $\{\gamma\}$ is the family of curves in $\tilde{D}-(|z| \leqq 1)$ separating $\tilde{\Gamma}$ from $|z|=1$. Let $\left\{\gamma_{1}\right\}$ be the family consisting of curves in the upper half of $\tilde{D}-(|z| \leqq 1)$ connecting $(1, \infty)-\bigcup_{n=1}^{\infty}\left[b_{n-1}, a_{n}\right]$ with $(-\infty,-1)-\bigcup_{n=1}^{\infty}\left[-a_{n},-b_{n-1}\right]$. Let $\left\{\gamma_{1}^{\prime}\right\}$ be its subfamily consisting of curves whose end points are symmetric with respect to the origin. Then, by (VIII), No. 9,

$$
\lambda\left\{\gamma_{1}^{\prime}\right\} \geqq \lambda\left(\gamma_{1}\right\}=\lambda\{\gamma\} / 2>0 .
$$

Consider the region $\Delta=(|\zeta|<\infty)-\bigcup_{n=1}^{\infty}\left[b_{n=1}^{2}, a_{n}^{2}\right]$ and its boundary component $(\zeta=\infty)$. Let $\left\{\gamma^{*}\right\}$ be the family of curves in $\Delta-(|\zeta| \leqq 1)$ separating $\infty$ from $|\zeta| \leqq 1$. By making use of the mapping $\zeta=z^{2}$, we can immediately see that $\lambda\left\{\gamma^{*}\right\}=\lambda\left\{\gamma_{1}^{\prime}\right\}$ and, therefore, $(\zeta=\infty)$ is an unstable boundary component of $\Delta$.

The mapping

$$
\zeta=T(z)=r^{2} e^{i \theta} \quad\left(z=r e^{i \theta}\right)
$$

is quasi-conformal and maps $D$ onto $\Delta, z=\infty$ onto $\zeta=\infty$. Since weakness is preserved under quasi-conformal mappings (No. 18), $\Gamma$ is unstable.

REMARK. Using the same method, we can also prove Theorem 10 when the intervals are distributed on $k$ half-lines $r e^{i 2 \pi \nu / k}(0 \leqq r<\infty)$, $\nu=0,1, \cdots, k$.

\section{Criteria for arbitrary regions.}

30. Let $D$ be a plane region such that $\Gamma=\{\infty\}$ is a boundary component. If $D$ is contained in another region discussed in preceding sections and $\{\infty\}$ is its unstable boundary component, then, by the definition of instability, $\Gamma$ is an unstable boundary component of $D$.

If such a condition is not satisfied, the following criterion may be applicable. It is a simple generalization of (ii) of Theorem 7, and we omit the proof.

Theorem 11. Let $D$ be a region such that $0 \in D$ and $\Gamma=\{\infty\}$ is a boundary component. $\Gamma$ is unstable if there exists a sequence $\left\{C_{n}\right\}_{n=1}^{\infty}$ of components of $\partial D-\Gamma$ satisfying the following conditions:

(i) For a doubly connected region $A \subset D$ separating 0 from $\infty$, there exists a number $n$ such that $A$ separates $C_{n}$ from $C_{n+1}$.

(ii) For every $n$, there exist points $a_{n} \in C_{n}$ and $b_{n} \in C_{n+1}$ such that $\left|a_{n}-b_{n}\right|=\operatorname{dist}\left(C_{n}, C_{n+1}\right)$, 


$$
\lim _{n \rightarrow \infty} \frac{b_{n}}{a_{n}}=1
$$

and

$$
\sum_{n=1}^{\infty} \frac{1}{\log \frac{1}{\left|\left(b_{n} \mid a_{n}\right)-1\right|}}<\infty .
$$

31. This criterion is not a necessary condition for instability. This is apparent from the following

EXAMPLE 6. Consider the closed sets

$$
\begin{aligned}
E_{n}=\left\{z ; n^{2}+1 \leqq|z| \leqq(n+1)^{2},\right. & \left.|\arg z| \leqq \pi-\varepsilon_{n}\right\}, \\
0<\varepsilon_{n}<\pi, & n=1,2, \cdots .
\end{aligned}
$$

If $\varepsilon_{n}(n=1,2, \cdots)$ are taken sufficiently small, then $\Gamma=\{\infty\}$ is an unstable boundary component of $D=(|z|<\infty)-\bigcup_{n=1}^{\infty} E_{n}$. It does not satisfy the assumption of Theorem 11 .

Proof. For an arbitrary subsequence $\left\{C_{n}\right\}_{n=1}^{\infty}$ of $\left\{E_{n}\right\}_{n=1}^{\infty}$ and every choice of $a_{n}$ and $b_{n}$,

$$
\sum_{n=1}^{\infty} \frac{1}{\log \frac{1}{\left|\left(b_{n} \mid a_{n}\right)-1\right|}} \geqq \frac{1}{2} \sum_{n=1}^{\infty} \frac{1}{\log n}=\infty .
$$

Therefore, the assumption of Theorem 11 is not satisfied.

In order to show the instability of $\Gamma$, consider the following cross cuts of $D$ :

$$
\begin{aligned}
& \alpha_{n}: \Re z=0,(n+1)^{2} \leqq \Im z \leqq(n+1)^{2}+1, \\
& \beta_{n}:|z|=(n+1)^{2},|\arg z| \leqq \pi-\varepsilon_{n}, \\
& \beta_{n}^{\prime}:|z|=(n+1)^{2}+1,|\arg z| \leqq \pi-\varepsilon_{n+1}, \\
& (n=1,2, \cdots) \text {. }
\end{aligned}
$$

Let $\delta_{n}$ be the extremal distance between $\alpha_{n}$ and $\beta_{n} \cup \beta_{n}^{\prime}$ with respect to the region $(n+1)^{2}<|z|<(n+1)^{2}+1$. It is possible to take $\varepsilon_{n}$ and $\varepsilon_{n+1}$ so small that $\delta_{n}>n^{2}(n=1,2, \cdots)$. Let $\{\gamma\}_{n}$ be the family consisting of closed curves in $D-(|z| \leqq 1)$ separating $\Gamma$ from $|z| \leqq 1$ and passing through $\alpha_{n}$. Let $\left\{\gamma_{1}\right\}_{n} \subset\{\gamma\}_{n}$ be the subfamily of curves contained in $(n+1)^{2}<|z|<(n+1)^{2}+1$ and put $\left\{\gamma_{2}\right\}_{n}=\{\gamma\}_{n}-\left\{\gamma_{1}\right\}_{n}$. By (VI), No. 9 , 


$$
\frac{1}{\lambda\{\gamma\}_{n}} \leqq \frac{1}{\lambda\left\{\gamma_{1}\right\}_{n}}+\frac{1}{\lambda\left\{\gamma_{2}\right\}_{n}}
$$

Since $n^{2}<\delta_{n} \leqq \lambda\left\{\gamma_{2}\right\}_{n}$ and $2 \pi / \lambda\left\{\gamma_{1}\right\}_{n}=\log \left(1+1 /(n+1)^{2}\right)$, we get

$$
\frac{1}{\lambda\{\gamma\}_{n}} \leqq \frac{1}{2 \pi} \log \left(1+\frac{1}{(n+1)^{2}}\right)+\frac{1}{n^{2}}
$$

if $n$ is sufficiently large, and, therefore, $\sum_{n=1}^{\infty} 1 / \lambda\{\gamma\}_{n}$ converges.

To apply Theorem 3 , take $A_{1}, A_{2}, \cdots, A_{k}$. Then evidently

$$
\sum_{\nu=1}^{k} \bmod A_{\nu} \leqq \sum_{n=1}^{\infty} 1 / \lambda\{\gamma\}_{n}<\infty
$$

and we conclude that $\Gamma$ is unstable.

32. Finally, for the sake of completeness, we shall present a wellknown sufficient condition for weakness. For a bounded doubly connected region $A$, we have that $\bmod A \geqq \log (1+(\pi d / 4 l))$. Here $d$ is the distance between the components of $\partial A$ and $l$ is the infimum of the lengths of closed curves which separate the components of $\partial A$ and whose distance from $\partial A$ is $\geqq d / 2$ (Sario [15], Meschkowsky [11]). Therefore we get immediately from Theorem 3 the following result (Meschkowsky [11], Savage [19]):

THEOREM 12. Let $D$ be a plane region containing the point $z=0$ and such that $\Gamma=\{\infty\}$ is a boundary component. Suppose there exists a sequence of doubly connected regions $A_{n} \subset D-(|z| \leqq \varepsilon)(n=1,2, \cdots)$ with the following properties:

(i) The $A_{n}$ are mutually disjoint,

(ii) $A_{n}$ separates $\Gamma$ from $|z| \leqq \varepsilon(n=1,2, \cdots)$ and also separates $A_{n-1}$ from $A_{n+1}(n=2,3, \cdots)$,

(iii)

$$
\sum_{n=1}^{\infty} d_{n} / l_{n}=\infty
$$

Then $\Gamma$ is a weak boundary component of $D$.

On applying this theorem, we obtain

ExAmple 7 (Denneberg [5]). Let $D$ be a region such that $\Gamma=\{\infty\}$ is the only accumulating boundary component. If there exist numbers $\alpha>0$ and $\beta<\infty$ such that the distance between every pair components of $\partial D-\Gamma$ is $\geqq \alpha$ and the diameter of every component of $\partial D-\Gamma$ is $\leqq \beta$, then $\Gamma$ is weak.

ExAmple 8 (Cf. Wagner [24]). Let \&s be the group of transforma- 
tions $z^{\prime}=z+m \omega+n \omega^{\prime}(m, n=0, \pm 1, \pm 2, \cdots)$ and let $E_{0}$ be a closed set contained in the interior of the fundamental parallelogram of (B). Then $\Gamma=\{\infty\}$ is a weak boundary component of the region $D=$ $(|z|<\infty)-\mathbf{U}_{T \in \mathscr{S}} T\left(E_{0}\right)$.

\section{Criteria For Strength AND Instability}

In this chapter we shall discuss Problem $B$, No. 4. For simplicity we mean by a boundary continuum a boundary component of a region which is a continuum containing more than one point.

\section{Strong boundary components.}

33. If $\Gamma$ is an isolated boundary continuum of $D$, i.e., if there exists an open set $U$ such that $\Gamma \subset U$ and $U \cap(\partial D-\Gamma)=\phi$, then $\Gamma$ is evidently strong. More generally,

Theorem 13. A boundary continuum $\Gamma$ of a region $D$ is strong if there exists a disk $U$ such that $U \cap \Gamma \neq \phi$ and $U \cap(\partial D-\Gamma)=\phi$.

This theorem is also almost trivial. To prove it rigorously, we shall use the following

LEMMA 3. Let $\Delta$ be a simply connected region which is a proper subset of $(|\zeta|<1)$. Map 4 conformally onto the upper half-plane. Then the image $E$ of $\overline{\partial \Delta \cap(|\zeta|<1)}$ is a set which does not belong to the class $N_{D}{ }^{5)}$

The proof is easy and we omit it. It may appear plausible that $E$ contains an interval. That this is however not so has been remarked by Koebe (see Radó [13], p. 2, Bemerkung). We can even see that the condition of Lemma 3 is necessary and sufficient.

Proof of Theorem 13. Map a component $\Delta$ of $U \cap D$ onto the upper half-plane by $\varphi$ and let $E$ be the image of $\Gamma \cap \bar{\Delta}$. By Lemma $3 E \notin N_{D}$ and, therefore, $E$ is of positive measure (Ahlfors and Beurling [2]). If $\Gamma$ is unstable, a univalent function $f(z)$ transforms $\Gamma$ to a point. Therefore, the univalent function $f \circ \mathcal{P}$ on the upper half-plane takes a constant boundary value on $E$, contrary to the well-known theorem of $\mathrm{F}$. and M. Riesz.

REMARK 1. In this case, $R(\Gamma)<\infty$ and we can also use Theorem 1 to conclude that $\Gamma$ is strong. To prove the finiteness of $R(\Gamma)$, we apply Theorem 5. Take a component $V$ of $U \cap D$. It is easy to find

${ }^{5}$ A compact set $E$ is said to belong to the class $N_{D}$ if $E^{c}$ does not admit a function with a finite Dirichlet integral. 
a simply connected region $\Delta$ such that $\Delta \subset D, V \subset \Lambda$ and $(|z| \leqq \varepsilon) \subset \Delta$. Since the set $E \notin N_{D}$ is of positive capacity (Ahlfors and Beurling [2]), $\lambda\{\gamma\}_{\varepsilon}<\infty$ by Lemma 3 and (XI), No. 10 .

REMARK 2. Because of this theorem, we may consider from now on only the case where every point of $\Gamma$ is an accumulation point of $\partial D-\Gamma$.

34. We shall now give two other kinds of examples of strong boundary components which do not satisfy the condition of Theorem 13 .

EXAMPLe 7. Let $D$ be a radial slit disc $|z|<a$ in the sense of No. 3 and let $\Gamma=(|z|=a)$. If the arguments of the slits form a set of measure $\mu$ less than $2 \pi$, then $R(\Gamma)<\infty$ and, consequently, $\Gamma$ is strong.

In fact, we can easily obtain the estimate

$$
\lambda\{\gamma\}_{\varepsilon} \leqq\{\log (a / \varepsilon)\} /(2 \pi-\mu)<\infty .
$$

35. EXAMPLE 8. Let $\left\{c_{n}\right\}_{n=1}^{\infty}$ be a sequence of numbers such that $0<c_{n} \leqq \pi / 2^{n+1}$. Put $r_{n}=1-1 /(n+1)$ and let

$$
\begin{gathered}
s_{n}^{k}=\left\{z ;|z|=r_{n}, \frac{\pi(k-1)}{2^{n}}+c_{n} \leqq \arg z \leqq \frac{\pi k}{2^{n}}-c_{n}\right\} \\
\left(k=1,2, \cdots, 2^{n+1} ; n=1,2, \cdots\right) .
\end{gathered}
$$

$\Gamma=(|z|=1)$ is a boundary continuum of the circular slit disc $D=$ $(|z|<1)-\bigcup_{n, k} s_{n}^{k}$. If $\underline{\lim }_{n \rightarrow \infty} c_{n} 2^{n}>0$, then $R(\Gamma)<\infty$ and therefore, $I^{\prime}$ is strong.

Proof. Clearly it is sufficient to give the proof for $c_{n} 2^{n}=\delta>0$. For simplicity, we choose $\delta=\pi / 4$, i.e., $c_{n}=\pi / 2^{n+2}$. In order to show the finiteness of $R(\Gamma)$, we map $D$ quasi-conformally onto the radial slit $\operatorname{disc} \Delta=(|w|<1)-\mathrm{U}_{n, k} \sigma_{n}^{k}$, where

$$
\begin{gathered}
\sigma_{n}^{k}=\left\{w ; r_{n} e^{-c_{n} / 2} \leqq|w| \leqq r_{n} e^{c_{n} / 2}, \arg w=\frac{\pi(2 k-1)}{2^{n+1}}\right\} \\
\left(k=1,2, \cdots, 2^{n+1} ; n=1,2, \cdots\right) .
\end{gathered}
$$

Consider the doubly connected regions

$$
\begin{aligned}
A_{z}=\{z & \left.-1<\Re z<1,-\frac{1}{2}<\Im z<\frac{1}{2}\right\} \\
& -\left\{z ;-\frac{1}{2} \leqq \Re z \leqq \frac{1}{2}, \Im z=0\right\}
\end{aligned}
$$

and

$$
\begin{aligned}
A_{w}=\{w ; & \left.-1<\Re w<1,-\frac{1}{2}<\Im w<\frac{1}{2}\right\} \\
& -\left\{w ; \Re w=0,-\frac{1}{4} \leqq \Im w \leqq \frac{1}{4}\right\}
\end{aligned}
$$


It is not difficult to map $A_{a}$ quasi-conformally onto $A_{w}$ by a function which is of class $C^{1}$ in $A_{z}$ and is the identity mapping on the outer periphery of $A_{z}$.

In our region $D$, consider the quadrilaterals

$$
\begin{gathered}
Q_{n}^{k}=\left\{z ; r_{n} e^{-c_{n}}<|z|<r_{n} e^{c_{n}}, \frac{\pi(k-1)}{2^{n}}<\arg z<\frac{\pi k}{2^{n}}\right\} \\
\left(k=1,2, \cdots, 2^{n+1} ; n=1,2, \cdots\right) .
\end{gathered}
$$

They are mutually disjoint and all $Q_{n}^{k}-s_{n}^{k}$ and $Q_{n}^{k}-\sigma_{n}^{k}$ are conformally equivalent to $A_{z}$ and $A_{w}$, respectively. Therefore, we can contruct the mapping $w=T_{n}^{k}(z)$ of $Q_{n}^{k}-s_{n}^{k}$ onto $Q_{n}^{k}-\sigma_{n}^{k}$ which is the identity mapping on $\partial Q_{n}^{k}$ and whose maximal dilatation $K$ depends neither on $k$ nor on $n$. Then

$$
w=T(z)= \begin{cases}T_{n}^{k}(z) & \text { in } Q_{n}^{k}-s_{n}^{k}\left(k=1,2, \cdots, 2^{n+1} ; n=1,2, \cdots\right) \\ z & \text { in } D-\bigcup_{n, k} Q_{n}^{k}\end{cases}
$$

is a qussi-conformal mapping of $D$ onto $\Delta$ such that $T(T)=(|w|=1)=\Gamma^{\prime}$.

Since $\Delta$ belongs to the case of Example $7, R\left(\Gamma^{\prime} ; \Delta\right)<\infty$, and, by Theorem $5, \lambda\left\{\gamma^{\prime}\right\}_{\varepsilon}<\infty$. Here $\gamma^{\prime}$ is a rectifiable curve in $\Delta-(|w| \leqq \varepsilon)$ connecting $|w|=\varepsilon$ with $\Gamma^{\prime}$. It is furthermore assumed that $\gamma^{\prime}$ is a union of a countable number of analytic arcs clustering nowhere in $\Delta$ (cf. Remark, No. 15). On $D$, we have the corresponding family $\{\gamma\}$ : and, by (IV), No. 15, $\lambda\{\gamma\}_{\varepsilon} \leqq K \lambda\left\{\gamma^{\prime}\right\}_{\varepsilon}<\infty$. Therefore, by Theorem 5, $R(\Gamma)<\infty$ and $\Gamma$ is strong.

35. We continue to consider Example 8. If $c_{n}$ decreases sufficiently fast, then $R(\Gamma)=\infty$. In fact, let $\left\{\gamma_{n}\right\}_{\varepsilon}$ be the subfamily of $\{\gamma\}_{\varepsilon}$ which consists of curves passing through the arc $\left\{z ; z=r_{n},|\arg z| \leqq c_{n}\right\}$. By (VI), No. 9, $\lambda\{\gamma\}_{\varepsilon} \geqq \lambda\left\{\gamma_{n}\right\}_{\varepsilon} / 2^{n+1}$ and, By Lemma 1, No. 13,

$$
\lambda\left\{\gamma_{n}\right\}_{\varepsilon} \sim \frac{1}{2 \pi} \log \frac{1}{c_{n}} \quad(n \rightarrow \infty) .
$$

For this reason $R\left(I^{\prime}\right)=\infty$ if, for instance, $c_{n}=\exp \left(-2^{2 n}\right)$. However, it is unknown in this case whether $\Gamma$ is strong or unstable.

\section{Unstable boundary continua.}

37. As in No. 21, let $\left\{\Omega_{n}\right\}_{n=1}^{\infty}$ be a defining sequence of $\Gamma$ and let $0 \in G_{n}=D-\Omega_{n} \uparrow D$. Consider the function $w=f_{n}(z)$ maximizing the functional $m(f)$ in $\mathfrak{F}_{F_{n}}$ on $G_{n}$ (No. 19). We may assume that $\left\{f_{n}(z)\right\}_{n=1}^{\infty}$ converges to a univalent function $w=f(z)$.

In the following case, $R(\Gamma)=\infty$ implies that $f(\Gamma)=\{\infty\}$ : 
Theorem 14. Let $D$ be a region containing $z=0$ and let $\Gamma$ be a boundary continum. Suppose that

(i) $D$ is symmetric with respect to the lines

$$
l_{\nu}: r e^{\nu \pi / 2 \kappa}(-\infty<r<\infty), \nu=1,2, \cdots, 2^{k}
$$

for some integer $k \geqq 0$, and

(ii) every component of $\partial D-\Gamma$ intersects at least one $l_{\nu}$.

Then $\Gamma$ is strong if and only if $R(\Gamma)<\infty$.

Proof. We may assume that each $G_{n}$ is symmetric with respect to all the $l_{\nu}$. By the uniqueness of $f_{n}(z)$ (No. 19), we can immediately see that $f_{n}(z)$ and, a fortiori, $f(D)$ are symmetric about these lines. As has been shown by Strebel [22], $f(\partial D-\Gamma)$ consists of radial segments. By the assumption $f(\partial D-\Gamma)$ is contained in $\bigcup_{\nu=1}^{2^{k}} l_{i}$.

Now assume that $f(\Gamma) \neq\{\infty\}$. If $f(\Gamma) \subset \bigcup_{\nu=1}^{2^{k}} l_{\nu} \cup\{\infty\}$, then $f(\Gamma) \cap l_{\nu}$ is a line segment which does not meet $f(\partial D-\Gamma)$, so that $R(\Gamma)<\infty$ by Remark 1, No. 33. If $f(\Gamma) \not \subset \bigcup_{\nu=1}^{2 k} l_{\nu} \cup\{\infty\}$ there exists a sector $S$ bounded by two neighboring $l_{\nu}$ 's such that $S \cap f(\Gamma)$ does not intersect $f(\partial D-\Gamma)$ and we have $R(\Gamma)<\infty$. Consequently, the strength of $\Gamma$ implies that $R(\Gamma)<\infty$.

38. We can find many examples of unstable boundary continua belonging to this category, e.g., as follows:

ExAMPle 9. Consider the region

$$
D=(|z| \leqq \infty)-\Gamma-\bigcup_{k=1}^{\infty}\left(s_{k}^{+} \cup s_{k}^{-} \cup_{\sigma_{k}^{+}}^{+} \cup_{\sigma k}^{-}\right),
$$

where

$$
\begin{aligned}
& \Gamma=\{z ;-1 \leqq \Re z \leqq 1, \mathfrak{\Im} z=0\}, \\
& s_{k}^{+}=\left\{z ; 1+\frac{1}{2 k+1} \leqq \Re z \leqq 1+\frac{1}{2 k}, \Im z=0\right\},
\end{aligned}
$$

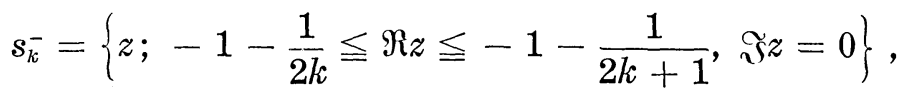

$$
\begin{aligned}
& \sigma_{k}^{ \pm}=\left\{z ;-1 \leqq \Re z \leqq 1, \Im z=\frac{ \pm 1}{k}\right\} \cdot
\end{aligned}
$$

Since every point on $\Gamma$, except \pm 1 , is inaccessible, $R(\Gamma)=\infty$ by $\left(X^{\prime}\right)$, No. 10. From this and from Theorem 14, we infer that $\Gamma$ is an unstable boundary continuum of $D$.

39. Meschkowsky [11] has proved that a region satisfying certain 
metric conditions can be mapped conformally onto a region bounded by circles or points in such a way that the image of a preassigned boundary continuum is a point. This case is also an example of an unstable boundary continuum.

40. The following example belongs to this category but does not necessarily satisfy Meschkowsky's conditions. Moreover, the function $f(z)=\lim _{n \rightarrow \infty} f_{n}(z)$ of No. 37 does not transform $\Gamma$ to a point.

EXAMPLE 10. Let $I=\{z ;-1 \leqq \Re z \leqq 1, \mathfrak{\Im} z=0\}$ and let

$$
I^{\prime}=\{z ; \Re z=0,-1 \leqq \Im z \leqq 1\} \text {. }
$$

Choose a sequence $\left\{c_{k} ; k= \pm 1, \pm 2, \cdots\right\}$ such that

$$
c_{-k}=-c_{k}, c_{1}>c_{2}>\cdots \downarrow 0 \text {, }
$$

and let

$$
\begin{aligned}
s_{k}^{0}: z & =r e^{i c_{k}} & & (1 /|k| ! \leqq r \leqq 1), \\
s_{k}^{\pi / 2}: z & =r e^{i\left(c_{k}+\pi / 2\right)} & & (1 /|k| ! \leqq r \leqq 1), \\
s_{k}^{\pi}: z & =r e^{i\left(c_{k}+\pi\right)} & & (1 /|k| ! \leqq r \leqq 1), \\
s_{k}^{-\pi / 2}: z & =r e^{i\left(c_{k}-\pi / 2\right)} & & (1 /|k| ! \leqq r \leqq 1),
\end{aligned}
$$

where $k= \pm 1, \pm 2, \cdots$. Then $\Gamma=I \cup I^{\prime}$ is an unstable boundary continuum of the region

$$
D=(|z| \leqq \infty)-\Gamma-\bigcup_{\substack{k=-\infty \\ k \neq 0}}^{\infty}\left(s_{k}^{0} \cup s_{k}^{\pi / 2} \cup s_{k}^{\pi} \cup s_{k}^{-\pi / 2}\right) .
$$

In fact, $D$ can be mapped onto a region such that $f(\Gamma)$ is a point and every component of $f(\partial D-I)$ is a circle. For the proof, map the region

$$
(|z|) \leqq \infty)-\bigcup_{\substack{k=-n \\ k \neq 0}}^{\infty}\left(s_{k}^{0} \cup s_{k}^{\pi / 2} \cup s_{k}^{\pi} \cup s_{k}^{-\pi / 2}\right)
$$

conformally onto a region bounded by $8 n$ circles; we may require that the mapping function $w=f^{(n)}(z)$ has the expansion $z+b_{n} / z+\cdots$ near $z=\infty(n=1,2, \cdots)$. The existence and the uniquess of such a mapping are well known. A suitable subsequence of $\left\{f^{(n)}(z)\right\}_{n=1}^{\infty}$ converges to a univalent function $w=f(z)$. We can easily prove that every component of $f(\partial D-\Gamma)$ is a circle (see, e.g., Meschkowsky [11]). In what follows we shall show that $f(\Gamma)=\{0\}$.

First we remark that $R(\Gamma)=\infty$, because every point on $\Gamma$, except $0, \pm 1, \pm i$, is inaccessible (cf. $\left(X^{\prime}\right)$, No. 10). Second, $D$ and, therefore, 
$f(D)$ are symmetric with respect to the following four lines: $l_{0}=$ (real axis), $l_{\pi / 4}=(\Re z=\Im z), l_{\pi / 2}=$ (imaginary axis), and $l_{-\pi / 4}=(\Re z=-\Im z)$.

The component $f(\Gamma)^{*}$ of $f(D)^{c}$ corresponding to $f(\Gamma)$ is a compact connected set which contains the point $w=0$ and is symmetric about these four lines.

The component $f\left(s_{k}^{\beta}\right)^{*}$ of $D^{c}(\beta=0, \pm \pi / 2, \pi ; k= \pm 1, \pm 2, \cdots)$ is a disk, which we denote by

$$
\Delta_{k}^{\beta}:\left|w-a_{k}^{\beta}\right| \leqq \rho_{k} .
$$

The radius $\rho_{k}$ does not depend on $\beta$ because of the symmetry. Furthermore,

$$
\lim _{k \rightarrow \infty} \rho_{k}=0 ;
$$

in fact, all the $\Delta_{k}^{\beta}$ cluster to $f(\Gamma)^{*}$, so that the sum $8 \pi \sum_{k=1}^{\infty} \rho_{k}^{2}$ of their areas converges.

Consider a quadrilateral

$$
Q_{k}=\left\{z ; \frac{1}{k !}<|z|<\frac{1}{(k-1) !}, c_{k}<\arg z<\frac{\pi}{2}-c_{k}\right\},
$$

which connects $s_{k}^{0}$ with $s_{-k}^{\pi / 2}(k=1,2, \cdots)$. The extremal distance between $s_{k}^{0}$ and $s_{-k}^{\pi / 2}$ with respect to $D$ does not exceed

$$
\bmod Q_{k}=\frac{(\pi / 2)-2 c_{k}}{\log k}
$$

Let $L_{k}$ be the infimum of lengths of curves in $f(D)$ connecting $\Delta_{k}^{0}$ with $\Delta_{-k}^{\pi / 2}$. Then

$$
\frac{L_{k}^{2}}{\mu U} \leqq \frac{(\pi / 2)-2 c_{k}}{\log k} \rightarrow 0
$$

where $\mu U$ expresses the area of a bounded open set $U$ containing $f(\Gamma)^{*}$. For this reason and by virtue of (17) and (18), we have

$$
\lim _{k \rightarrow \infty}\left|a_{k}^{0}-a_{-k}^{\pi / 2}\right| \leqq \lim _{k \rightarrow \infty}\left(L_{k}+2 \rho_{k}\right)=0 \text {. }
$$

It follows, by symmetry, that $\left\{a_{k}^{0}\right\}_{k=1}^{\infty}$ and $\left\{a_{-k}^{\pi / 2}\right\}_{k=1}^{\infty}$ cluster to $l_{\pi / 4}$ in the first quadrant. From this and again from the symmetry, we see that the set $H$ of all accumulation points of $\alpha_{k}^{\beta}(\beta=0, \pm \pi / 2, \pi ; k= \pm 1$, $\pm 2, \cdots)$ is contained in $l_{\pi / 4} \cup l_{-\pi / 4}$. Evidently it is symmetric about $l_{0}$ and $l_{\pi / 2}$, and $H \subset f(\Gamma)^{*}$.

Next we shall show that $H=\{0\}$. Suppose that $H$ contains a point $w_{0}=p e^{i \pi / 4}(p>0)$. Then there must exist a point $q e^{i \pi / 4} \in H(0 \leqq q<p)$. For otherwise $H$ would consist of four points: $H=\left\{p e^{i \theta} ; \theta= \pm \pi / 4, \pm 3 \pi / 4\right\}$. 
Then all but a finite number of components of $f(\partial D-\Gamma)$ in the first quadrant would be contained in $\left|w-p e^{i \pi / 4}\right|<p / 4$. Since $w_{0}$ and 0 are contained in $f(\Gamma)^{*}$ and $f(\Gamma)^{*}$ is a continuum, $f(\Gamma)$ would have a "free" subset as in Theorem 13. But the reasoning of Remark 1, No. 33, shows that this property of $f(\Gamma)$ contradicts the fact that $R(\Gamma)=\infty$ and, therefore, $q e^{i \pi / 4} \in H$ exists. Take a subsequence $\left\{k_{j}\right\} \subset\{k\}$ such that

$$
\lim _{j \rightarrow \infty} a_{k_{j}}^{0}=\lim _{j \rightarrow \infty} a_{-k_{j}}^{\pi / 2}=q e^{i \pi / 4}
$$

Then

$$
L_{k_{j}}+2 \rho_{k_{j}} \geqq \frac{p-q}{2}>0
$$

for sufficiently great $j$, contrary to (17) and (18). Consequently, $w_{0}$ does not exist and $H=\{0\}$.

Finally, if $f(\Gamma)^{*} \supsetneqq H$, then $f(\Gamma)$ would again have a "free" subset, contrary to the fact that $R(\Gamma)=\infty$. We conclude that $f(\Gamma)^{*}=\{0\}$.

41. Transform the region $D$ by $\zeta=1 / z$ and, for simplicity, denote the image again by $D$. For the sequence $G_{n} \uparrow D$ of No. 37, we take

$$
\begin{aligned}
G_{n}=\left(|z|<n !+c_{n+1}\right) & \cap D \\
-\bigcup_{n=1}^{3}\left\{z ; 1-c_{n+1}\right. & \leqq|z|, \frac{h \pi}{2}-\frac{c_{n}+c_{n+1}}{2} \leqq \arg z \\
& \left.\leqq \frac{h \pi}{2}+\frac{\left.c_{n}+c_{n+1}\right\}}{2}\right\},
\end{aligned}
$$

$n=1,2, \cdots$, and consider the extremal function $f_{n}(z)$. We shall show:

If $c_{k}=-c_{-k}$ decreases sufficiently fast (e.g., $c_{k}=e^{-k !}$ ), then $\lim _{n \rightarrow \infty} f_{n}(z)=z$ uniformly on every compact set in $D$.

In order to prove this, we estimate the Dirichlet integral of $\log \left|f_{n}(z) / z\right|$ over $\Delta=(|z| \leqq 1 / 2)$ :

$$
\begin{aligned}
& D_{\Delta}\left(\log \left|f_{n}(z)\right|-\log |z|\right) \leqq D_{G_{n}}\left(\log \left|f_{n}(z)\right|-\log |z|\right) \\
= & \int_{\partial G_{n}}\left(\log \left|f_{n}\right| \cdot d \arg f_{n}-\log |z| \cdot d \arg f_{n}\right. \\
& \left.-\log \left|f_{n}\right| \cdot d \arg z+\log |z| \cdot d \arg z\right) \\
= & \int_{\partial G_{n}}\left(\log \left|f_{n}\right| \cdot d \arg f_{n}-2 \log \left|f_{n}\right| \cdot d \arg z\right. \\
& +\log |z| \cdot d \arg z) \\
= & 2 \pi \log R\left(\Gamma_{n} ; G_{n}\right)-2 \log R\left(\Gamma_{n} ; G_{n}\right) \int_{F_{n}} d \arg z \\
& +\int_{\Gamma_{n}} \log |z| d \arg z \leqq 2 \pi\left\{\log n !-\log R\left(\Gamma_{n} ; G_{n}\right)\right\} .
\end{aligned}
$$


To estimate the last term, we shall use the relation $\log R\left(\Gamma_{n} ; G_{n}\right)=$ $\lim _{\varepsilon \rightarrow 0}\left(\log \varepsilon+2 \pi \lambda\{\gamma\}_{\varepsilon}^{(n)}\right)$, where the sequence is increasing (No. 22). Here $\{\gamma\}_{\varepsilon}^{(n)}$ is the family of curves in $G_{n}-(|z| \leqq \varepsilon)$ connecting $\Gamma_{n}$ with $|z|=\varepsilon$. We take the closed disks

$$
\begin{aligned}
& \Delta_{n}^{h}:\left|z-e^{i \pi \hbar / 2}\right| \leqq c_{n}, \\
& \Delta_{n}^{\prime h}:\left|z-n ! e^{i \pi h / 2}\right| \leqq n ! c_{n},
\end{aligned}
$$

$h=0,1,2,3 ; n=1,2, \cdots$. Let $\left\{\gamma_{1}\right\}_{\varepsilon}^{(n)} \subset\{\gamma\}_{\varepsilon}^{(n)}$ be the family of curves connecting $|z|=\varepsilon$ with $\bigcup_{h, n} \Delta_{n}^{h} \cup \Delta_{n}^{\prime h}$ and put $\left\{\gamma_{2}\right\}_{\varepsilon}^{(n)}=\{\gamma\}_{\varepsilon}^{(n)}--\left\{\gamma_{1}\right\}_{\varepsilon}^{(n)}$. By (VI), No. 9,

$$
\frac{1}{\lambda\{\gamma\}_{\varepsilon}^{(n)}} \leqq \frac{1}{\lambda_{1}}+\frac{1}{\lambda_{2}} \quad\left(\lambda_{\nu}=\lambda\left\{\gamma_{\nu}\right\}_{\varepsilon}^{(n)}, \nu=1,2\right),
$$

or

$$
\lambda\{\gamma\}_{\varepsilon}^{(n)} \geqq \lambda_{2}-\frac{\lambda_{2}^{2}}{\lambda_{1}}
$$

It is evident that

$$
\frac{1}{2 \pi-8 c_{n}} \log \frac{n !+c_{n}}{\varepsilon} \geqq \lambda_{2} \geqq \frac{1}{2 \pi} \log \frac{n !}{\varepsilon} .
$$

Therefore,

$$
\log R\left(\Gamma_{n} ; G_{n}\right) \geqq \log \varepsilon+2 \pi \lambda\{\gamma\}_{\varepsilon}^{(n)} \geqq \log n !-2 \frac{\lambda_{2}^{2}}{\lambda_{1}},
$$

whence

$$
D_{\Delta}\left(\log \left|f_{n}(z)\right|-\log |z|\right) \leqq 4 \pi^{2} \frac{\lambda_{2}^{2}}{\lambda_{1}} .
$$

If $c_{n}$ is taken sufficiently small, then $\lim _{n \rightarrow \infty} \lambda_{2}^{2} / \lambda_{1}=0$. For instance, if $c_{n}=e^{-n !}$, we have $\lambda_{1} \sim(8 \cdot n !) / \pi(n \rightarrow \infty)$ by Lemma 1 , No. 13, and $\lambda_{2}^{2} / \lambda_{1} \rightarrow 0$. In such a case, $\lim _{n \rightarrow \infty} D_{\Delta}\left(\log \left|f_{n}(z)\right|-\log |z|\right)=0$ and we conclude that $\lim _{n \rightarrow \infty} f_{n}(z)=z$ uniformly on each compact set in $D$.

Consequently $R(\Gamma)=\infty$ for our region, but $\lim _{n \rightarrow \infty} f_{n}(z)$ does not transform $\Gamma$ to a point.

\section{REFERENCES}

1. L. V. Ahlfors, On quasiconformal mappings, J. Analyse Math. 3 (1953/54), 1-58, 207-208.

2. L. V. Ahlfors, and A. Beurling, Conformal invariants and function-theoretic null-sets, Acta Math. 83 (1950), 101-129.

3. L. V. Ahlfors, and L. Sario, Riemann surfaces, Princeton University Press, to appear.

4. C. Constantinescu, Sur la comportement d'une fonction analytique à la frontière d'une surface de Riemann, C. R. Acad. Sci. Paris, 245 (1957), 1995-1997. 
5. H.Denneberg, Konforme Abbildung einer Klasse unendlich-vielfach zusammenhängender schlichter Bereiche auf Kreisbereiche, Ber. Verh. Sächs. Akad. Wiss. Leipzig. Math-Nat. K1. 84 (1932), 331-352.

6. H. Grötzsch, Über die Verzerrung bei schlichter konformen Abbildung mehrfach zusammenhängender schlichter Bedeiche, I. Ibid. 81 (1929), 38-47. II. 217-221.

7. —, Eine Bemerkung zum Koebeschen Kreisnormierungsprinzip, Ibid. 87 (1935), 319-324.

8. J. Hersch, Longueurs extrémales et théorie des fonctions, Comment. Math. Helv. 29 (1955), 301-337.

9. - Contribution à la théorie des fonctions pseudo-analytiques, Ibid. 30 (1956), $1-19$.

10. M. Jurchescu, Modulus of a boundary component, Pacific J. Math. 8 (1958), 791-809.

11. H. Meschkowsky, Über die konforme Abbildung gewisser Bereiche von unendlich hohem Zusammenhang auf Vollkreisbereiche, I. Math. Ann. 123 (1951), 392-405. II. Ibid. 124 (1952), 178-181.

12. A. Pfluger, Extremallängen und Kapazität, Comment. Math. Helv. 29 (1955), 120-131.

13. T. Radó, Über eine nicht fortsetzbare Riemannsche Mannigfaltigkeit, Math. Z. 20 (1924), 1-6.

14. E. Rengel, Existenzbeweise für schlichte Abbildungen mehrfach zusammenhängender Bereiche auf gewisse Normalbereiche, Jber. Deutsch Math. Verein. 45 (1935), 83-87.

15. L. Sario, Über Riemannsche Flächen mit hebbarem Rànd, Ann. Acad. Sci. Fenn. Ser. A. I. 50 (1948), $79 \mathrm{pp}$.

16. - Capacity of the boundary and of a boundary component, Ann. Math. $\mathbf{5 9}$ (1954), 135-144.

17. - Stability problems on boundary components, Proc. Conference Anal. Func., Princeton, 1957. To appear.

18. - Strong and weak boundary components, J. Analyse Math. 5 (1958), 389-398. 19. N. Savage, Weak boundary components of an open Riemann surface, Duke Math. J. 24 (1957), 79-96.

20. K. Strebel, Eine Ungleichung für extremale Längen, Ann. Acad. Sci. Fenn. Ser. A. I. 90 (1951), 8 pp.

21. - Über das Kreisnormierungsproblem der konformen Abbildung, Ibid. 101 (1951), $22 \mathrm{pp}$.

22. - Die extremale Distanz zweier Enden einer Riemannschen Fläche, Ibid. 179 (1955), $22 \mathrm{pp}$.

23. O. Teichmüller, Untersuchungen über konforme und quasikonforme Abbildung, Deutsche Math. 3 (1938), 621-678.

24. R. Wagner, Ein Kontaktproblem der konformen Abbildung, J. Reine Angew. Math. 196 (1956), 99-132.

25. V. Wolontis, Properties of conformal invariants, Amer. J. Math. 74 (1952), 587-606.

University of California, Los ANgeles, California

AND

TOKYo InSTItUTE of TEChNOLOGY, JAPAN 


\title{
PACIFIC JOURNAL OF MATHEMATICS
}

\section{EDITORS}

\author{
David Gilbarg \\ itanford University \\ itanford, California \\ F. H. Brownele \\ Jniversity of Washington \\ ieattle 5 , Washington
}

\author{
A. L. Whiteman
}

University of Southern California Los Angeles 7. California

L. J. Paige

University of California

Los Angeles 24, California

ASSOCIATE EDITORS
ミ. F. BECKENBACH
E. HEWITT
M. OHTSUKA
E. SPANIER
r. M. CHERRY
A. HORN
H. L. ROYDEN
E. G. STRAUS
). DERRY
L. NACHBIN
M. M. SCHIFFER
F. WOLF

\section{SUPPORTING INSTITUTIONS}

JNIVERSITY OF BRITISH COLUMBIA
ZALIFORNIA INSTITUTE OF TECHNOLOGY
JNIVERSITY OF CALIFORNIA
UONTANA STATE UNIVERSITY
JNIVERSITY OF NEVADA
NEW MEXICO STATE UNIVERSITY
JREGON STATE COLLEGE
JNIVERSITY OF OREGON
JSAKA UNIVERSITY
JNIVERSITY OF SOUTHERN CALIFORNIA

STANFORD UNIVERSITY

UNIVERSITY OF TOKYO

UNIVERSITY OF UTAH

WASHINGTON STATE COLLEGE

UNIVERSITY OF WASHINGTON

AMERICAN MATHEMATICAL SOCIETY CALIFORNIA RESEARCH CORPORATION HUGHES AIRCRAFT COMPANY

SPACE TECHNOLOGY LABORATORIES

NAVAL ORDNANCE TEST STATION

Printed in Japan by Kokusai Bunken Insatsusha

(International Academic Printing Co., Ltd.), Tokyo, Japan 


\section{Pacific Journal of Mathematics}

\section{Vol. 10, No. $1 \quad$ September, 1960}

Richard Arens, Extensions of Banach algebras................... 1

Fred Guenther Brauer, Spectral theory for linear systems of differential equations....................................... 17

Herbert Busemann and Ernst Gabor Straus, Area and normality ......... 35

J. H. Case and Richard Eliot Chamberlin, Characterizations of tree-like

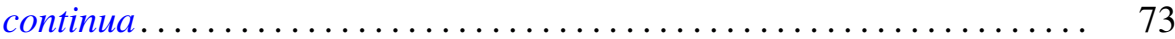

Ralph Boyett Crouch, Characteristic subgroups of monomial groups...... 85

Richard J. Driscoll, Existence theorems for certain classes of two-point boundary problems by variational methods ................. 91

A. M. Duguid, A class of hyper-FC-groups ................. 117

Adriano Mario Garsia, The calculation of conformal parameters for some

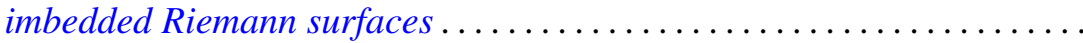

Irving Leonard Glicksberg, Homomorphisms of certain algebras of measures ........................................... 167

Branko Grünbaum, Some applications of expansion constants . . . . . . . . . 193

John Hilzman, Error bounds for an approximate solution to the Volterra integral equation ................................... 203

Charles Ray Hobby, The Frattini subgroup of a p-group ............... 209

Milton Lees, von Newmann difference approximation to hyperbolic

equations...................................... 213

Azriel Lévy, Axiom schemata of strong infinity in axiomatic set theory .... 223

Benjamin Muckenhoupt, On certain singular integrals ............... 239

Kotaro Oikawa, On the stability of boundary components ............. 263

J. Marshall Osborn, Loops with the weak inverse property .............. 295

Paulo Ribenboim, Un théorème de réalisation de groupes réticulés ....... 305

Daniel Saltz, An inversion theorem for Laplace-Stieltjes transforms ....... 309

Berthold Schweizer and Abe Sklar, Statistical metric spaces ........... 313

Morris Weisfeld, On derivations in division rings ................ 335

Bertram Yood, Faithful ${ }^{*}$-representations of normed algebras ........... 345 